\title{
Methods of Blood Flow Modelling
}

\author{
N. Bessonov ${ }^{1}$, A. Sequeira ${ }^{2}$ S. Simakov ${ }^{3,4}$ Yu. Vassilevskii ${ }^{3,4}$ V. Volpert ${ }^{5}$ * \\ ${ }^{1}$ Institute of Problems of Mechanical Engineering, Russian Academy of Sciences \\ 199178 Saint Petersburg, Russia \\ ${ }^{2}$ Departamento de Matemática and CEMAT/IST Instituto Superior Técnico, Universidade de Lisboa \\ Av. Rovisco Pais 1, 1049-001 Lisboa, Portugal \\ ${ }^{3}$ Moscow Institute of Physics and Technology, 9 Instituskii Lane, 141707 Dolgoprudny, Russia \\ ${ }^{4}$ Institute of Numerical Mathematics RAS, 7 Gubkina St., 119333 Moscow, Russia \\ ${ }^{5}$ Institut Camille Jordan, UMR 5208 CNRS, University Lyon 1, 69622 Villeurbanne, France
}

\begin{abstract}
This review is devoted to recent developments in blood flow modelling. It begins with the discussion of blood rheology and its non-Newtonian properties. After that we will present some modelling methods where blood is considered as a heterogeneous fluid composed of plasma and blood cells. Namely, we will describe the method of Dissipative Particle Dynamics and will present some results of blood flow modelling. The last part of this paper deals with onedimensional global models of blood circulation. We will explain the main ideas of this approach and will present some examples of its application.
\end{abstract}

Keywords and phrases: blood rheology, shear thinning, viscoelasticity, dissipative particle dynamics, global circulation

Mathematics Subject Classification: 92C35, 76A10, 76M12, 76Z05, 70-08, 35L40

\section{Blood Rheology}

Rheology is the study of deformation and flow of a material. We say that a body has been deformed if its shape or size has been altered due to the action of appropriate forces. If the degree of deformation changes continuously with time, the body is considered to be flowing. This section deals with hemorheology: the science of deformation and flow of blood and its formed elements. This field includes investigations of both macroscopic blood properties using rheometric experiments as well as microscopic properties in vitro and in vivo. Hemorheology also encompasses the study of the interactions among blood components and between these components and the endothelial cells that line blood vessels.

Advances in the field of hemorheology are based on the evidence that they might be the primary cause of many cardiovascular diseases. In fact, hemorheological aberrations can easily be considered as a result (or an indicator) of insufficient circulatory function. Basically, pathologies with hematological origin like leukemia, hemolytic anemia, thalassemia or pathologies associated with the risk factors of thrombosis and atherosclerosis like myocardial infarction, hypertension, strokes or diabetes are mainly related to disturbances of local homeostasis. Therefore, the mathematical and numerical study constitutive models

*Corresponding author. E-mail: volpert@math.univ-lyon1.fr

(C) EDP Sciences, 2016 
that can capture the rheological response of blood over a range of flow conditions is ultimately recognized as an important tool for clinical diagnosis and therapeutic planning (see e.g. $[38,83]$ ).

To better interpret and analyze the experimental data on blood it is helpful to turn to the literature on the rheology of particle suspensions. For rigid particles, a vast amount of published literature exists (see e.g. [115]). However, the study of suspensions of multiple, interacting and highly deformable particles such as blood, has received less attention and presents a challenge for both theoretical and computational fluid dynamicists.

In this section we present a brief overview of the rheological properties of blood, including its most relevant non -Newtonian characteristics and discuss constitutive models introduced to capture one or more of these properties.

\subsection{Blood components}

Blood is a concentrated suspension of several formed cellular elements, red blood cells (RBCs or erythrocytes), white blood cells (WBCs or leukocytes) and platelets (thrombocytes), in an aqueous polymeric and ionic solution, the plasma, composed of $93 \%$ water and $3 \%$ particles, namely, electrolytes, organic molecules, numerous proteins (albumin, globulins and fibrinogen) and waste products. Plasma's central physiological function is to transport these dissolved substances, nutrients, wastes and the formed cellular elements throughout the circulatory system. Normal erythrocytes are biconcave discs with a mean diameter of 6 to $8 \mu \mathrm{m}$ and a maximal thickness of $1.9 \mu \mathrm{m}$. The average volume of an erythrocyte is 90 $\mu m^{3}$ ([26] ). Their number per cubic millimetre of blood is approximately 5 to $6 \times 10^{6}$ and they represent approximately 40 to $45 \%$ by volume of the normal human blood and more than $99 \%$ of all blood cells. The first percentage is called hematocrit. The primary function of erythrocytes is to transport oxygen and carbon dioxide. Leukocytes are roughly spherical and much larger than erythrocytes, but they exist in a smaller number in blood: their diameter ranges between 6 and $17 \mu \mathrm{m}$ and there are approximately 7 to $11 \times 10^{3}$ per cubic millimetre in a normal adult. Leukocytes are subdivided into granulocytes $(65 \%)$, lymphocytes $(30 \%)$, monocytes $(5 \%)$ and natural killer cells. Granulocytes are further subdivided into neutrophils (95\%), eosinophils (4\%) and basophils (1\%). The leukocytes play a vital role in fighting infection and thus are able to migrate out of the blood vessels and into the tissues. Thrombocytes are small discoid non-nucleated cell fragments, much smaller than erythrocytes and leukocytes (approximately 2 to $3 \mu \mathrm{m}^{3}$ in volume). Thrombocytes are a vital component of the blood clotting mechanism. The total volume concentration of leukocytes and thrombocytes is only about $1 \%$.

Blood cells are continually produced by the bone marrow over a human's life. For example, erythrocytes have an average lifetime of 120 days and the body must produce about $3 \times 10^{9}$ new erythrocytes for each kilogram of body weight every day. Due to ageing and rupturing they must be constantly replaced (see e.g. [66]).

\subsection{Non-Newtonian properties of blood}

The mechanical properties of blood should be studied by considering a fluid containing a suspension of particles. A fluid is said to be Newtonian if it satisfies the Newton's law of viscosity (the shear stress is proportional to the rate of shear and the viscosity is the constant of proportionality). Blood plasma, which consists mostly of water, is a Newtonian fluid. However, the whole blood has complex mechanical properties which become particularly significant when the particles size is much larger, or at least comparable, with the lumen size. In this case, which happens at the microcirculation level (in the small arterioles and capillaries) blood cannot be modelled has a homogeneous fluid and it is essential to consider it as a suspension of blood cells (specially RBCs) in plasma. The presence of the blood cellular elements and their interactions leads to significant changes in the blood rheological properties and reliable measurements need to be performed to derive appropriate microstructural models.

Otherwise, depending on the size of the blood vessels and the flow behaviour, it is approximated as a Navier-Stokes fluid or as a non-Newtonian fluid. Here we assume that all macroscopic length and time scales are sufficiently large compared to length and time scales at the level of the individual erythrocyte 
so that the continuum hypothesis holds. Thus the models presented here are not appropriate in the capillary network. For an overview of hemorheology in the microcirculation we refer the reader to the review article of Popel and Johnson [110].

\subsubsection{Viscosity of blood}

In general blood has higher viscosity than plasma, and when the hematocrit rises, the viscosity of the suspension increases and the non-Newtonian behaviour of blood becomes more relevant, in particular at very low shear rates. The apparent viscosity increases slowly until a shear rate less than $1 s^{-1}$, where it rises markedly [26]. The reason for this is that at low shear rates the erythrocytes have the ability to form a primary aggregate structure of rod shaped stacks of individual cells called rouleaux, that align to each other and form a secondary structure consisting of branched three-dimensional (3D) aggregates [118]. It has been experimentally observed that rouleaux will not form if the erythrocytes have been hardened or in the absence of fibrinogen and globulins (plasma proteins) [30]. In fact, suspensions of erythrocytes in plasma demonstrate a strong non-Newtonian behaviour whereas when they are in suspension in physiological saline (with no fibrinogen or globulins) the behaviour of the fluid is Newtonian $[37,86]$. For standing blood subjected to a shear stress lower than a critical value, these 3D structures can form and blood exhibits yield stress and resists to flow until a certain force is applied. This can happen only if the hematocrit is high enough. The existence of yield stress for blood will be discussed below (see 1.2.3).

At moderate to high shear rates, RBCs are dispersed in the plasma and the properties of the blood are influenced by their tendency to align and form layers in the flow, as well as to their deformation. The effect of RBC deformability on the viscosity of suspensions was clearly shown in [30].

For shear rates above $400 s^{-1}$, the RBCs lose their biconcave shape, become fully elongated and are transformed into ellipsoids with major axes parallel to the flow direction. The tumbling of the RBCs is absent, there are almost no collisions, and their contours change according to the tank-trading motion of the cells membranes about their interior. The apparent viscosity decreases and this becomes more evident in smaller than in larger vessels. This happens with vessels of internal diameter less than $1 \mathrm{~mm}$ and it is even more pronounced in vessels with a diameter of 100 to $200 \mu \mathrm{m}$. The geometric packing effects and radial migration of RBCs can act to lower the hematocrit adjacent to the vessel wall and contribute to decrease the blood viscosity. This is known as the Fähraeus -Lindqvist effect. Plasma skimming is another effect that results in diminishing the viscosity when blood flows into small lateral vessels compared with the parent vessel.

As a consequence of this behaviour we can say that one of the non-Newtonian characteristics of blood is the shear thinning viscosity. This happens in small size vessels or in regions of stable recirculation, like in the venous system and parts of the arterial vasculature where geometry has been altered and RBC aggregates become more stable, like downstream a stenosis or inside a saccular aneurysm. However, in most parts of the arterial system, blood flow is Newtonian in normal physiological conditions.

Figure 1 displays the shear thinning behaviour of whole blood as experimentally observed by [30]. Each of these data points represents an equilibrium value obtained at a fixed shear rate.

As a first step towards the macroscopic modelling of blood flow we consider the simplest constitutive model for incompressible viscous fluids based on the assumption that the extra stress tensor is proportional to the symmetric part of the velocity gradient,

$$
\tau=2 \mu D
$$

where $\mu$ is the (constant) viscosity and $D=\left(\nabla u+\nabla u^{T}\right) / 2$ is the rate of deformation tensor. The substitution of $\tau$ in the equations of the conservation of linear momentum and mass (or incompressibility condition) for isothermal flows given by 


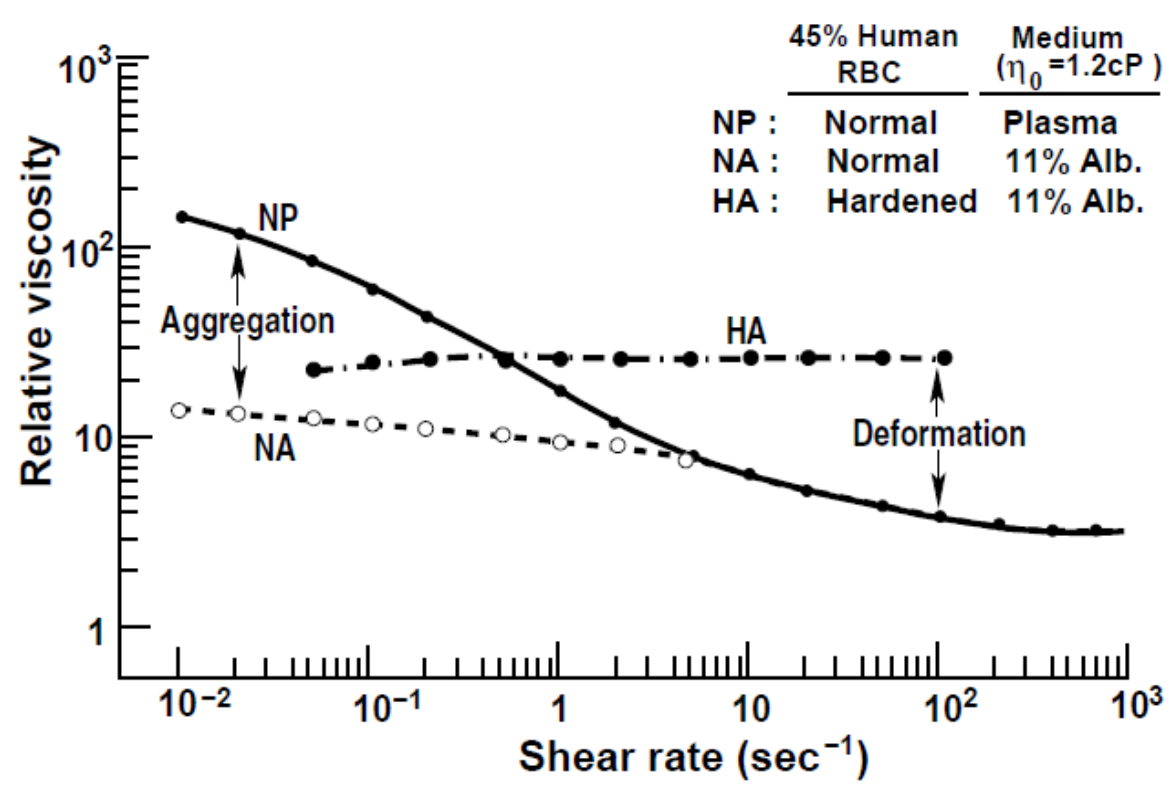

FiguRE 1. Variation of the relative viscosity as a function of the shear rate for normal $\mathrm{RBC}$ in heparinised plasma (NP), normal RBC in albumin-Ringer solution (NA) and hardened RBCs in albumin-Ringer solution (HA) at a temperature of $37^{\circ} \mathrm{C}$, hematocrit $H_{t}=45 \%$ using a Couette viscometer (reproduced from [30]).

$$
\begin{aligned}
& \rho \frac{\partial u}{\partial t}+\rho(u \cdot \nabla) u=-\nabla p+\nabla \cdot \tau \\
& \nabla \cdot u=0
\end{aligned}
$$

leads to the well-known Navier-Stokes equations for an incompressible viscous fluid. Here $u$ and $p$ denote the blood velocity and pressure, with $t \geq 0, \rho$ is the blood density and $\tau$ is the extra-stress tensor. System (1.2) is closed with appropriate initial and boundary conditions.

As already discussed, this set of equations is commonly used to describe blood flow in healthy arteries. However, under certain experimental or physiological conditions, particularly at low shear rates, blood exhibits relevant non-Newtonian characteristics and more complex constitutive models need to be used. In this case, we require a more general constitutive equation relating the state of stress to the rate of deformation which satisfies invariance requirementts [11]. One of the simplest is the special class of Reiner-Rivlin fluids, called generalised Newtonian fluids, for which

$$
\tau=2 \mu(\dot{\gamma}) D
$$

where $\dot{\gamma}$ is the shear rate (a measure of the rate of deformation) defined by

$$
\dot{\gamma}=\sqrt{2 \operatorname{tr}\left(D^{2}\right)}=\sqrt{-4 \mathrm{II}_{\mathrm{D}}}
$$

and $\mu(\dot{\gamma})$ is a shear dependent function. Here $\mathrm{II}_{\mathrm{D}}$ denotes the second principal invariant of the tensor $D$, given by 


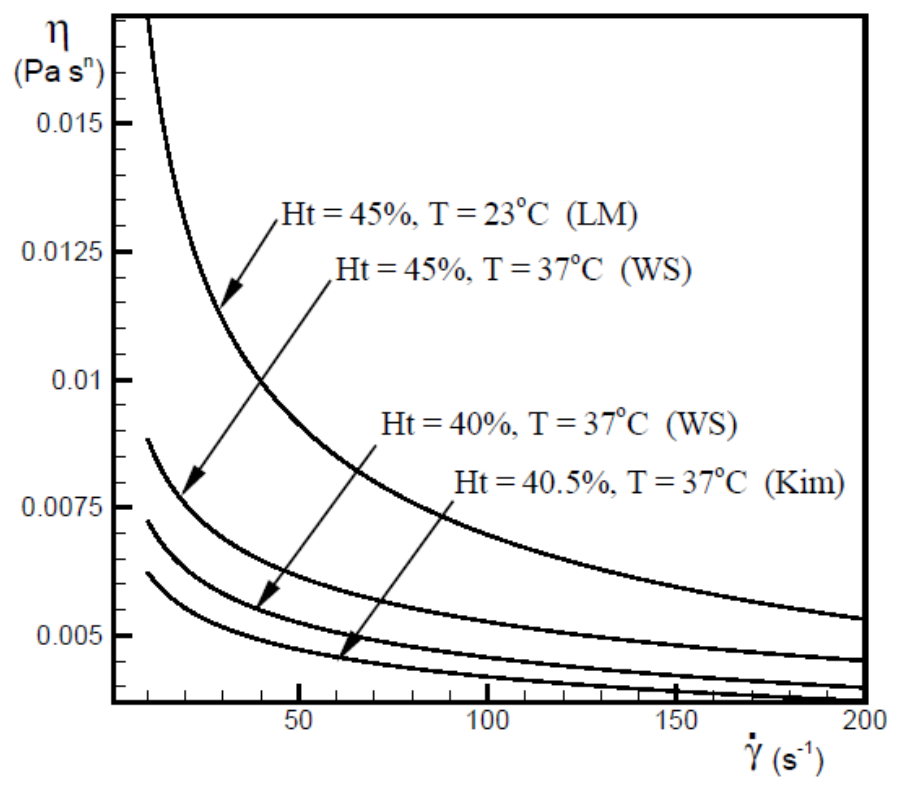

FiguRE 2. Comparison of viscosity functions $\mu(\dot{\gamma})$ for extensions of the power-law model (1.6) using material constants obtained by curve fit to experiments.

$$
\mathrm{II}_{\mathrm{D}}=1 / 2\left((\operatorname{tr} \mathrm{D})^{2}-\operatorname{tr} \mathrm{D}^{2}\right)
$$

(for isochoric motions $\mathrm{II}_{\mathrm{D}}$ is not a positive constant).

A simple example of a generalised Newtonian fluid is the power-law fluid, for which the viscosity function is given by

$$
\mu(\dot{\gamma})=K \dot{\gamma}^{n-1},
$$

the positive constantts $n$ and $K$ being the power-law index and the consistency, respectively. This model includes, as a particular case, the constant viscosity fluid (Newtonian) when $n=1$. For $n<1$ it leads to a monotonic decreasing function of the shear rate (shear thinning fluid) and for $n>1$ the viscosity increases with shear rate (shear thickening fluid). The shear thinning power-law model is often used for blood, due to the analytical solutions easily obtained for its governing equations, but it predicts an unbounded viscosity at zero shear rate and zero viscosity when $\dot{\gamma} \rightarrow \infty$, which is unphysical.

One of the extensions of the power-law model is due to Walburn and Schneck [140] who considered the dependence of the viscosity on the hematocrit $\left(H_{t}\right)$ and total protein minus albumin (TPMA) in the constants $n$ and $K$, based on nonlinear regression analysis, and found

$$
K=C_{1} \exp \left(C_{2} H_{t}\right), \quad n=1-C_{3} H_{t} .
$$

Figure 2 shows a comparison of viscosity functions $\mu(\dot{\gamma})$ for the power-law model (1.6) using material constants given by Kim et al [69] (Kim) and Liepsch and Moravec [79] (LM) for human blood. Representative curves for the Walburn-Schneck model (WS) [140] with factors depending on the hematocrit are also shown. The viscosity functions obtained from [140] for $H_{t}=40 \%$ and [69] for $H_{t}=40.5 \%$ are 
quite close. In contrast, those in [69] and [79] for $H_{t}=45 \%$ are substantially different, likely due to the difference in the temperatures.

Viscosity functions with bounded and non-zero limiting values of viscosity can be written in the general form

$$
\mu(\dot{\gamma})=\mu_{\infty}+\left(\mu_{0}-\mu_{\infty}\right) F(\dot{\gamma})
$$

or, in non-dimensional form as

$$
\frac{\mu(\dot{\gamma})-\mu_{\infty}}{\mu_{0}-\mu_{\infty}}
$$

Here, $\mu_{0}$ and $\mu_{\infty}$ are the asymptotic viscosity values at zero and infinite shear rates and $F(\dot{\gamma})$ is a shear dependent function, satisfying the following natural limit conditions

$$
\lim _{\dot{\gamma} \rightarrow 0} F(\dot{\gamma})=1, \quad \lim _{\dot{\gamma} \rightarrow \infty} F(\dot{\gamma})=0 .
$$

Different choices of the function $F(\dot{\gamma})$ correspond to different models for blood flow, with material constants quite sensitive and depending on a number of factors including hematocrit, temperature, plasma viscosity, age of RBCs, exercise level, gender or disease state.

Table 1 summarises some of the most common generalised Newtonian models that have been considered in the literature for the shear dependent viscosity of whole human blood. Values for the material constants given in this table were obtained by Cho and Kensey[32] for a compilation of human and canine blood (Ht ranging from $33-45 \%$ ), using a nonlinear least squares analysis.

\begin{tabular}{lcc}
\hline Model & $\frac{\mu(\dot{\gamma})-\mu_{\infty}}{\mu_{0}-\mu_{\infty}}$ & Material constants for blood \\
\hline Powell-Eyring & $\frac{\sinh ^{-1}(\lambda \dot{\gamma})}{\lambda \dot{\gamma}}$ & $\lambda=5.383 \mathrm{~s}$ \\
Cross & $\frac{1}{1+(\lambda \dot{\gamma})^{m}}$ & $\lambda=1.007 s, m=1.028$ \\
Modified Cross & $\frac{1}{\left(1+(\lambda \dot{\gamma})^{m}\right)^{a}}$ & $\lambda=3.736 s, m=2.406, a=0.254$ \\
Carreau & $\left(1+(\lambda \dot{\gamma})^{2}\right)^{(n-1) / 2}$ & $\lambda=3.313 s, n=0.3568$ \\
Carreau-Yasuda & $\left(1+(\lambda \dot{\gamma})^{a}\right)^{(n-1) / a}$ & $\lambda=1.902 s, n=0.22, a=1.25$ \\
\hline
\end{tabular}

TABLE 1. Material constants for various generalised Newtonian models for blood with $\mu_{0}=0.056$ Pa.s, $\mu_{\infty}=0.00345$ Pa.s

In addition, we should point out that the viscosity of whole blood is strongly dependent on temperature and care must be taken when data is obtained from different sources. Merrill et al. [85] found the dependence of blood viscosity on temperature to be similar to that of water for temperatures ranging from $10^{0}$ to $40^{0} \mathrm{C}$ and shear rates from 1 to $100 \mathrm{~s}^{-1}$. A close variation is also reported for plasma viscosity (see also [28]).

\subsubsection{Viscoelasticity and thixotropy of blood}

Viscoelastic fluids are viscous fluids which have the ability to store and release energy. The viscoelasticity of blood at normal hematocrits is primarily attributed to the reversible deformation of the RBCs $3 \mathrm{D}$ 
microstructures [131]. Elastic energy is due to the properties of the RBC membrane which exhibits stress relaxation [42] and the bridging mechanisms within the 3D structure. Moreover, the experimental results of Thurston [129] imply that the relaxation time depends on the shear rate. The reader is referred to [131] for a review of the dependence of blood viscoelasticity on factors such as temperature, hematocrit and $\mathrm{RBC}$ properties. In view of the available experimental evidence, it is reasonable to develop non-Newtonian fluid models for blood that are capable of shear thinning and stress relaxation, with the relaxation time depending on the shear rate. To date, very little is known concerning the response of such fluids. In fact, viscoelastic properties are of relatively small magnitude and they have generally only been measured in the context of linear viscoelasticity. By shear rates of the order of $10 \mathrm{~s}^{-1}$ the elastic nature of blood is negligible as evidenced by a merging of the oscillatory and steady flow viscosities. However, there is a need to consider the finite viscoelastic behaviour of blood, if viscoelastic constitutive equations are used to model blood in the circulatory system.

A number of nonlinear viscoelastic constitutive models for blood are now available but because of their complexity we will avoid presenting the mathematical details here, providing instead a summary of the relevant literature. One of the simplest rate type models accounting for the viscoelasticity of blood is the Maxwell model

$$
\tau+\lambda_{1} \frac{\delta \tau}{\delta t}=2 \mu D
$$

where $\lambda_{1}$ is the relaxation time and $\delta(.) / \delta t$ stands for the so-called convected derivative, a generalisation of the material time derivative, chosen so that $\delta \tau / \delta t$ is objective under a superposed rigid body motion and the resulting second-order tensor is symmetric[114].

A more general class of rate type models, includes the Oldroyd-B models defined by

$$
\tau+\lambda_{1} \frac{\delta \tau}{\delta t}=2 \mu\left(D+\lambda_{2} \frac{\delta D}{\delta t}\right),
$$

where the material coefficient $\lambda_{2}$ denotes the retardation time and is such that $0 \leq \lambda_{2}<\lambda_{1}$. The Oldroyd type fluids can be considered as Maxwell fluids with additional viscosity. These models (1.8) contain the previous model (1.7) as a particular case. Thurston [129], was among the earliest to recognise the viscoelastic nature of blood and that the viscoelastic behaviour is less prominent with increasing shear rate. He proposed a generalised Maxwell model that was applicable to one dimensional flow simulations (see section 3) and observed later that, beyond a critical shear rate, the nonlinear behaviour is related to the microstructural changes that occur in blood [130]. Thurston's work was suggested to be more applicable to venous or low shear unhealthy blood flow than to arterial flows. Recently, a generalised Maxwell model related to the microstructure of blood, inspired on the behaviour of transient networks in polymers, and exhibiting shear thinning, viscoelasticity and thixotropy (defined below), has been derived by [101].

Other viscoelastic constitutive models of differential type, suitable for describing blood rheology have been proposed in the recent literature. The empirical three constant generalised Oldroyd -B model studied in [149] belongs to this class. It has been obtained by fitting experimental data in one dimensional flows and generalising such curve fits to three dimensions. This model captures the shear thinning behaviour of blood over a large range of shear rates but it has some limitations, since the relaxation times do not depend on the shear rate, which does not agree with experimental observations. The model developed by Anand and Rajagopal [9] in the general thermodynamic framework stated in [113] includes relaxation times depending on the shear rate and gives good agreement with experimental data in steady Poiseuille and oscillatory flows.

Another important property of blood is its thixotropic behaviour, essentially due to the fact that the formation of the three-dimensional microstructure and the alignment of the RBCs are not instantaneous. Essentially, we refer to thixotropy as the dependence of the material properties on the time over which shear has been applied. This dependence is due to the finite time required for the build-up and breakdown 
of the 3D microstructure, elongation and recovery of RBCs and the formation and breakdown of layers of the aligned RBCs [13].

\subsubsection{Yield stress of blood}

The behaviour of many fluids at low shear stress, including blood, has led researchers to believe in the existence of a critical value of stress below which the fluid will not flow. This critical stress level, called the yield value or yield, is typically treated as a constant material property of the fluid. An extensive description of methods for measuring yield stress is given in [96]. Reported values for the yield stress of blood have a great variation ranging from 0.002 to 0.40 dynes $/ \mathrm{cm}^{2}$, see e.g. [37]. This variation has been attributed to artifacts arising from interactions between the RBCs and surfaces of the rheometer as well as to the experimental methods used to measure the yield stress and the length of time over which the experiments are run [13]. Rather than treating the yield stress as a constant, it should be considered as a function of time and linked to thixotropy, as later proposed by other researchers [89].

Yield stress models can be useful to model blood flow in low shear rate regions. Yield stress materials require a finite shear stress $\tau_{Y}$ (the yield stress) to start flowing. A relatively simple, and physically relevant yield criterion is given by

$$
\sqrt{|\mathrm{II}|_{\tau}}=\tau_{Y}
$$

where $\mathrm{II}_{\tau}$ is the second invariant of the extra stress tensor, $\tau$ (see (1.5)). Therefore, for $\sqrt{|\mathrm{II}|}<\tau_{Y}$, the fluid will not flow.

The most common yield stress model for blood is the Casson model ([119]) which, in simple shear flow, has the form

$$
\begin{aligned}
& \sqrt{\left|\mathrm{II}_{\tau}\right|}<\tau_{Y} \Longrightarrow D=0 \\
& \sqrt{\left|\mathrm{II}_{\tau}\right|} \geq \tau_{Y} \Longrightarrow\left\{\begin{array}{l}
D=\frac{1}{2 \mu_{N}}\left(1-\frac{\sqrt{\tau_{Y}}}{\sqrt{\left|\mathrm{II}_{\tau}\right|}}\right)^{2} \tau \\
\tau=2\left(\sqrt{\mu_{N}}+\frac{\sqrt{\tau_{Y}}}{\sqrt[4]{4\left|\mathrm{II}_{\mathrm{D}}\right|}}\right)^{2} .
\end{array}\right.
\end{aligned}
$$

The Newtonian constitutive equation is a special case of (1.10) for $\tau_{Y}$ equal to zero, in which case, $\mu_{N}$ is the Newtonian viscosity. The Casson fluid behaves rigidly until (1.9) is satisfied, after which it displays a shear thinning behaviour.

Other yield stress models like Bingham or Herschel-Bulkley models are also used for blood (see e.g. [114]) as well as the constitutive model developed by Quemada [112] using an approach, with the apparent viscosity $\mu$ given by

$$
\mu=\mu_{F}\left(1-\frac{1}{2} \frac{k_{0}+k_{\infty} \sqrt{\dot{\gamma} / \dot{\gamma}_{c}}}{1+\sqrt{\dot{\gamma} / \dot{\gamma}_{c}}} \varphi\right)^{-2},
$$

where $\mu_{F}, \varphi$ and $\dot{\gamma}_{c}$ are the viscosity of the suspending fluid, the volume concentration of the dispersed phase and a critical shear rate, respectively.

As discussed above, the existence of a yield stress and its use as a material parameter is still nowadays a controversial issue, due to the sensitivity of yield stress measurements.

In summary, we can conclude that blood is generally a non-Newtonian fluid, which can however be regarded as a Newtonian fluid to model blood flow in arteries with diameters larger than $100 \mu \mathrm{m}$ where measurements of the apparent viscosity show that it ranges from 0.003 to 0.004 Pa.s and the typical Reynolds number is about 0.5 . 


\section{Particle methods}

In the previous section we discussed blood characteristics in the case where blood was considered as a homogeneous non-Newtonian fluid. An alternative approach is to describe it as a complex fluid that consists of blood plasma and blood cells. Blood plasma can be considered as Newtonian fluid and described by the Navier-Stokes equations or by particle methods. Blood cells can also be modeled with partial differential equations of continuum mechanics or they can be considered as ensemble of particles with various forces acting between them. In both cases, the interaction of cells with the fluid and between them should be taken into account.

Various particle methods are developed to model blood flows including Smoothed-Particle Hydrodynamics, Lattice Gas Automata, Lattice Boltzmann, Dissipative Particle Dynamics (DPD), and blood cells in flow, Cellular Potts Method or Subcellular Element Method. We will present in this section the DPD method and some of its applications to blood flow modelling. At the end of this section we will briefly discuss hybrid models where particle methods are coupled with partial differential equations.

\subsection{Dissipative particle dynamics}

Dissipative Particle Dynamics (DPD) is a mesoscale method where each particle describes a small volume of the simulated medium rather than an individual molecule $[45,47,59,65]$. Their interaction is determined by the conservative, dissipative and random forces acting between each two particles:

$$
\begin{aligned}
& \mathbf{F}_{i j}^{C}=F_{i j}^{C}\left(r_{i j}\right) \hat{\mathbf{r}}_{i j}, \\
& \mathbf{F}_{i j}^{D}=-\gamma \omega^{D}\left(r_{i j}\right)\left(\mathbf{v}_{i j} \cdot \hat{\mathbf{r}}_{i j}\right) \hat{\mathbf{r}}_{i j}, \\
& \mathbf{F}_{i j}^{R}=\sigma \omega^{R}\left(r_{i j}\right) \frac{\xi_{i j}}{\sqrt{\Delta t}} \hat{\mathbf{r}}_{i j},
\end{aligned}
$$

where $\mathbf{r}_{i}$ is the vector of position of the particle $i, \mathbf{r}_{i j}=\mathbf{r}_{i}-\mathbf{r}_{j}, r_{i j}=\left|\mathbf{r}_{i j}\right|, \hat{\mathbf{r}}_{i j}=\mathbf{r}_{i j} / r_{i j}$, and $\mathbf{v}_{i j}=\mathbf{v}_{i}-\mathbf{v}_{j}$ is the difference between velocities of two particles, $\Delta$ t is the time step in a simulation, $\gamma$ and $\sigma$ are coefficients which determine the strength of the dissipative and the random force respectively, while $\omega^{D}$ and $\omega^{R}$ are weight functions; $\xi_{i j}$ is a normally distributed random variable with zero mean, unit variance, and $\xi_{i j}=\xi_{j i}$. The conservative force is given by the equality

$$
F_{i j}^{C}\left(r_{i j}\right)= \begin{cases}a_{i j}\left(1-r_{i j} / r_{c}\right) & \text { for } r_{i j} \leq r_{c}, \\ 0 & \text { for } r_{i j}>r_{c},\end{cases}
$$

where $a_{i j}$ is the conservative force coefficient between particles $i$ and $j$, and $r_{c}$ is the cut-off radius. Each of the forces is applied to the fluid particles as well as to the particles constituting blood cell membranes.

The system will preserve its energy and maintain the equilibrium temperature if the following two relations are satisfied:

$$
\omega^{D}\left(r_{i j}\right)=\left[\omega^{R}\left(r_{i j}\right)\right]^{2}, \quad \sigma^{2}=2 \gamma k_{B} T,
$$

where $k_{B}$ is the Boltzmann constant and $T$ is the temperature. The weight functions are determined by the equality:

$$
\omega^{R}\left(r_{i j}\right)= \begin{cases}\left(1-r_{i j} / r_{c}\right)^{k} & \text { for } r_{i j} \leq r_{c} \\ 0 & \text { for } r_{i j}>r_{c}\end{cases}
$$


where $k=1$ for the original DPD method, but it can be also varied in order to change the dynamic viscosity of the simulated fluid [45]. The motion of particles is determined by Newton's second law of motion:

$$
\Delta \mathbf{r}_{i}=\mathbf{v}_{i} \Delta t, \quad \Delta \mathbf{v}_{i}=\frac{\Delta t}{m_{i}} \sum_{j \neq i}\left(\mathbf{F}_{i j}^{C}+\mathbf{F}_{i j}^{D}+\mathbf{F}_{i j}^{R}\right),
$$

where $m_{i}$ is the mass of the particle $i$.

Euler method or a modified version of the velocity-Verlet method [7,59], which is more accurate, can be used to integrate the equations (2.6). In the former,

$$
\begin{aligned}
\mathbf{v}_{i}^{n+1} & =\mathbf{v}_{i}^{n}+\frac{1}{m_{i}} \mathbf{F}_{i}\left(\mathbf{r}_{i}^{n}, \mathbf{v}_{i}^{n}\right) \Delta t, \\
\mathbf{r}_{i}^{n+1} & =\mathbf{r}_{i}^{n}+\mathbf{v}_{i}^{n+1} \Delta t,
\end{aligned}
$$

where indices $n$ and $n+1$ denote time steps, and

$$
\mathbf{F}_{i}=\sum_{j \neq i}\left(\mathbf{F}_{i j}^{C}+\mathbf{F}_{i j}^{D}+\mathbf{F}_{i j}^{R}\right)
$$

A two substep discretization is suggested in [15]:

$$
\begin{aligned}
& \mathbf{r}_{i}^{n+1}=\mathbf{r}_{i}^{n}+\mathbf{v}_{i}^{n} d t+\frac{1}{2} \mathbf{a}_{i}^{n} \Delta t^{2}, \quad \mathbf{v}_{i}^{n+\frac{1}{2}}=\mathbf{v}_{i}^{n}+\frac{1}{2} \mathbf{a}_{i}^{n} \Delta t \\
& \mathbf{a}_{i}^{n+1}=\frac{1}{m_{i}} \mathbf{F}_{i}\left(\mathbf{r}_{i}^{n+1}, \mathbf{v}_{i}^{n+\frac{1}{2}}\right), \quad \mathbf{v}_{i}^{n+1}=\mathbf{v}_{i}^{n+\frac{1}{2}}+\frac{1}{2} \mathbf{a}_{i}^{n+1} \Delta t
\end{aligned}
$$

where $a_{i}^{n}$ denotes the acceleration of the particle $i$ at the $n^{\text {th }}$ time step. Both methods give close results but the second one allows an essential increase of the time step. Characteristic values of parameters are as follows: $r_{c}=1 \cdot 10^{-6} \mathrm{~m}, a_{i, j}=1 \cdot 10^{-12} \mathrm{~N}, \gamma=1 \cdot 10^{-8} \mathrm{~kg} \cdot \mathrm{s} / \mathrm{m}^{4}, \sigma=1 \cdot 10^{-11} \mathrm{~kg} /\left(\mathrm{m}^{3} \cdot \sqrt{s}\right)$.

The DPD method and its applications to fluid mechanics are intensively studied $[45,48,59,65,117]$. In $[45,48]$ DPD simulation results are compared with the Navier-Stokes and Stokes equations for Couette, Poiseuille, square-cavity and triangular-cavity flow. One of the important question in DPD simulations of fluid motion concerns the boundary conditions $[48,108,136,137]$.

\subsection{Erythrocyte models}

The red blood cell (RBC) membrane consists of a lipid bilayer and spectrin network connected by transmembrane proteins [88]. It is practically incompressible and resistant to the change of surface area and planar shear deformation. It can be modelled as a two-dimensional network of particles $[15,62,63,63,64,97,138,139]$. They are connected by springs modelled by Hooke's law to form an irregular polyhedron with triangular faces. There are four forces acting on particles. One more force related to the DPD method will be introduced below. Let us recall that cell membrane is modelled as a mesh which consists of triangular elements. The first force appears when the sides of the triangles change their length. There are several sides converging to each node and each of these sides contributes to the total force $\mathrm{F}$. The force acting on the node $a$ from the node $b$ equals

$$
\mathbf{F}_{I}=k_{I}\left(1-\frac{l}{l_{0}}\right) \boldsymbol{\tau}
$$

where $l$ is the length of the edge between two vertices, $l_{0}$ is the equilibrium length, and $k_{I}$ is the stiffness coefficient, $\boldsymbol{\tau}$ is the unit vector directed from node $a$ to node $b$. Furthermore, the forces acting on the nodes $a$ and $b$ have equal absolute values and opposite directions. 
In order to describe the second force, let us introduce the control volume for each node of the mesh. The outer boundary of the control volume is a piece-wise linear closed curve. It crosses the centers of the sides of the triangles having given node as one of their vertices and their centers of mass. The mass of the control volume is concentrated at the node. The motion of this node (or particle) is determined by the sum of forces acting on it.

The second force is determined by compression or dilation of the membrane. In order to define it, in each triangle we introduce the pressure given by the formula:

$$
\mathbf{p}_{s}=k_{s}\left(1-\frac{s}{s_{0}}\right)
$$

where $s$ is the area of triangular element, $s_{0}$ is the equilibrium area, $k_{s}$ is the area expansion modulus. This pressure creates three forces in the plane of the triangle. They are perpendicular to the triangle sides and they are proportional to their lengths. These forces are applied to the vertices of the triangle.

Since the out-of-plane bending deformation is present in RBC behavior, bending springs are introduced. The bending moment is considered between each pair of neihgboring triangular elements in the following form:

$$
\mathbf{M}_{\mathbf{i}}=k_{M} \tan \left(\frac{\theta}{2}\right) l \boldsymbol{\tau}_{\boldsymbol{i}}
$$

where $\theta$ is the angle between neighbouring triangular elements, $k_{M}$ is the stiffness coefficient, $\boldsymbol{\tau}_{\boldsymbol{i}}$ is the unit vector co-directional with the common edge of two triangles and $l$ is this side's length [63]. Each triangle has the total moment from its three sides $\mathbf{M}_{\Sigma}=\mathbf{M}_{1}+\mathbf{M}_{2}+\mathbf{M}_{3}$. This moment determines three forces $\mathbf{F}_{\mathbf{i}}, i=1,2,3$ directed perpendicular to the plane of the triangle. In order to express them through the moment $\mathbf{M}_{\Sigma}$ the following system of equations should be solved:

$$
\left\{\begin{array}{l}
\mathbf{F}_{1}+\mathbf{F}_{2}+\mathbf{F}_{3}=0 \\
\mathbf{a}_{1} \times \mathbf{F}_{1}+\mathbf{a}_{2} \times \mathbf{F}_{2}+\mathbf{a}_{3} \times \mathbf{F}_{3}=\mathbf{M}_{\Sigma}
\end{array}\right.
$$

where

$$
\mathbf{a}_{1}=\mathbf{r}_{a}-\mathbf{R}, \mathbf{a}_{2}=\mathbf{r}_{b}-\mathbf{R}, \mathbf{a}_{3}=\mathbf{r}_{c}-\mathbf{R}, \mathbf{R}=\left(\mathbf{r}_{a}+\mathbf{r}_{b}+\mathbf{r}_{c}\right) / 3
$$

$\mathbf{r}_{a}, \mathbf{r}_{b}, \mathbf{r}_{c}$ are radius-vectors of the vertices of the triangle $a b c . \mathbf{F}_{\mathbf{i}}, i=1,2,3$ are the forces that act on the vertices of the triangle. All these forces are parallel to the unit vector $\mathbf{n}$ normal to the surface. The solution of the system (2.15) has the form:

$$
\left\{\begin{array}{l}
\mathbf{F}_{\mathbf{1}}=\frac{\left(\mathbf{a}_{\mathbf{2}}-\mathbf{a}_{3}\right) \cdot \mathbf{M}_{\Sigma}}{\mathbf{s}} \mathbf{n}, \\
\mathbf{F}_{\mathbf{2}}=\frac{\left(\mathbf{a}_{\mathbf{3}}-\mathbf{a}_{1}\right) \cdot \mathbf{M}_{\Sigma}}{\mathbf{s}} \mathbf{n}, \\
\mathbf{F}_{\mathbf{3}}=\frac{\left(\mathbf{a}_{1}-\mathbf{a}_{2}\right) \cdot \mathbf{M}_{\Sigma}}{s} \mathbf{n} .
\end{array}\right.
$$

So far, only membrane characteristics have been described, which alone does not ensure the RBC shape. One more force, which depends on the volume of the polyhedron is introduced:

$$
\mathbf{F}_{v}=k_{v}\left(1-\frac{v}{v_{0}}\right) s \mathbf{n}
$$

where $v$ is the polyhedron volume, $v_{0}$ is the equilibrium volume, and $k_{v}$ is the coefficient, $s$ is the area of triangular element, $S$ is the total surface area of the polyhedron, and $\mathbf{n}$ is the unit normal vector to this triangle. This force is proportional to the change of volume of the polyhedron. It is applied to the vertices of triangles in the direction normal to the surface.

Another erythrocyte model that combines the continuous description of an elastic body for surface elements and a spring model for the angle between these elements is suggested in [15]. The membrane is 
represented as a two-dimensional surface that consists of elastic triangular elements. Two submodels are considered, one with linear and another one with nonlinear elasticity. We will call the Model I the spring model described above, the linear surface element, Model II, and nonlinear surface element, Model III. Model III gives the best approximation of experimental results on erythrocyte deformation (Figure 3).

Let us also note that other blood cells can be modelled in a similar way. Contrary to erythrocytes, leucocytes contain nucleus. Because of that, they are less deformable and their shape is close to a sphere. This influences their behavior in blood flow.
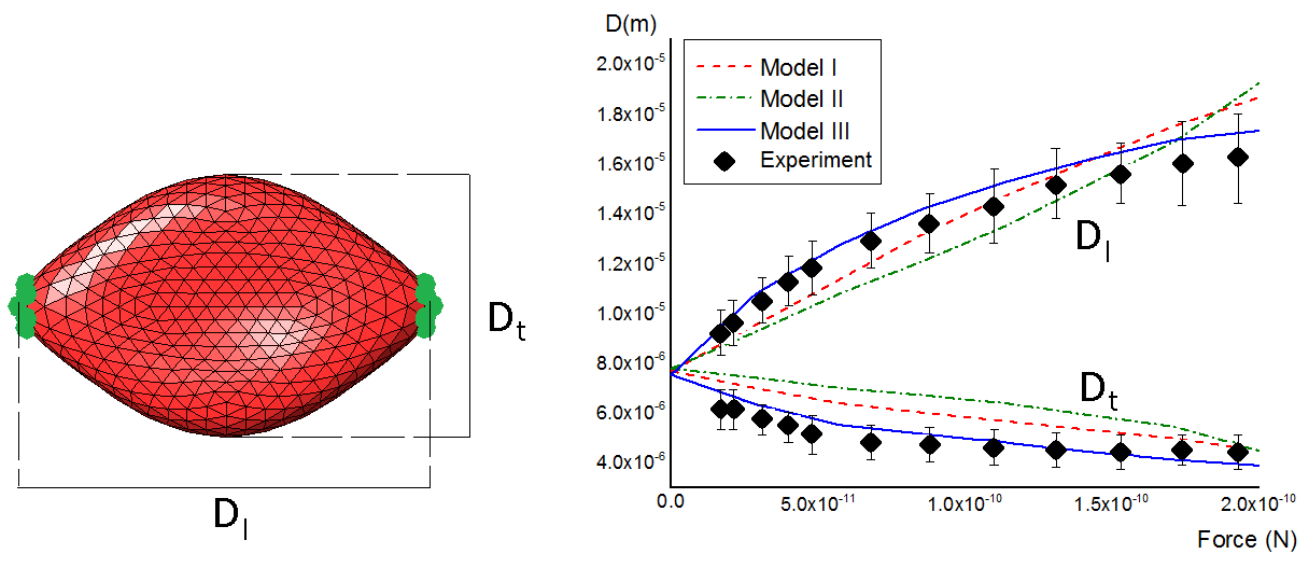

FiguRE 3. Modelling of the experiment on the stretching of erythrocyte (left). Comparison of experiments and numerical simulations for different models of erythrocyte (right). Reprinted with permission from [15].

\subsection{Blood flow modelling}

2.3.0.1. Stretching RBC. One of the experimental methods to determine mechanical properties of erythrocytes is stretching by optical tweezers. The experiment provides the values of transversal and longitudinal diameters of a cell under two stretching loads [127]. In order to determine parameters of our erythrocyte model, this experiment can be reproduced in numerical simulations $[15,47,63]$. Consider a $\mathrm{RBC}$ with the initial biconcave shape. We choose several nodes from the opposite sides and apply a force to them. We stretch the cell by a force with the values used in the experiment and measure the cell size in two directions. The values of parameters are chosen to fit the experimental curves. Figure 3 shows the scheme of the numerical experiment and comparison with experiments for the three models. Nonlinear surface element model (Model III) gives the best agreement with the experiment [15]. Other two models give approximately linear dependence of the diameters on force, and differ from the experimental dependence.

2.3.0.2. Cell distribution. Blood cell distribution in flow is important for their physiological functions. It is determined by their mechanical properties. Erythrocytes are concentrated more to the axis of the flow (Fähraeus -Lindqvist effect) and push platelets and leucocytes to the vessel walls. Examples of numerical simulations of blood flow with erythrocytes and platelets are shown in Figure 4 for different values of hematocrit [15]. The diameter of cylinder is $27 \mu \mathrm{m}$, its length is $100 \mu \mathrm{m}$ (only a part of it is shown).

Figure 5 (left) shows erythrocyte and platelet distributions in the cross section of cylinder for different values of $H_{t}[6,15]$. Erythrocyte concentration is greater near the axis, while platelets are localized near the wall. This effect is well-known from the experiments $[71,105,106,147,148]$. Velocity distribution in the cross section of the vessel is shown in Figure 5 (right). While the velocity profiles were parabolic in 

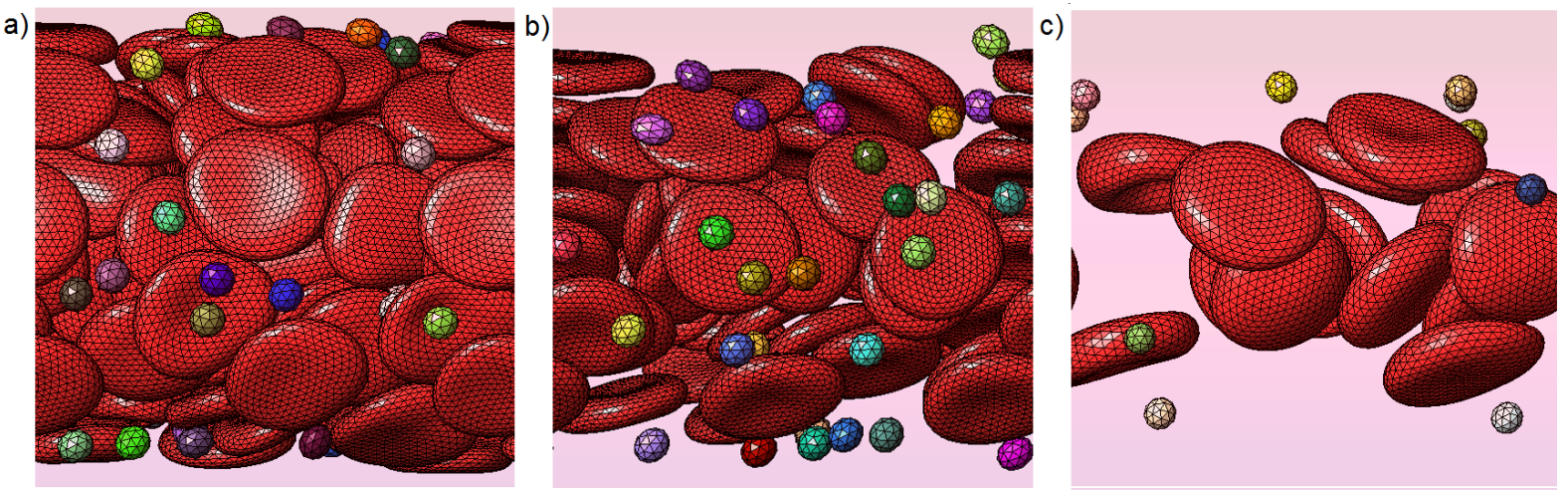

FiguRE 4. Snapshots of numerical simulations of blood flow with erythrocytes and platelets in the cylindrical channel for three different values of $H_{t}$ : a) $40 \%$, b) $20 \%$, c) $10 \%$. Blood plasma is modelled with the DPD method. It occupies the remaining volume of the channel (not shown). Reprinted with permission from [15].

simulations with only plasma particles, in simulations with erythrocytes the velocity profiles were flat in the middle of the vessel because of the high erythrocyte concentration. The influence of cells presence became stronger with increasing of $H_{t}$ value. These results are in good agreement with the experimental data $[56]$.

Platelets participate in blood coagulation, and their higher concentration near the blood vessel wall corresponds to their function. White blood cells (WBC) take part in the immune response. They move along the vessel wall [76] and stop at the inflammation cite due to the receptor activation at the surface of endothelial cells. WBC are less deformable than erythrocytes due to the presence of the nucleus, and they are pushed by erythrocytes to the vessel wall. This effect is also observed in numerical simulations [15].

\subsection{Blood coagulation}

Blood coagulation is an important physiological mechanism involving complex biochemical reactions in plasma and platelet aggregation. There are various approaches to model blood coagulation and clot growth. Concentrations of blood factors and of platelets can be described by reaction-diffusion-convection equations $[5,10,19,60,135]$. Individual cell model with cell interaction in the process of clot growth are studied in $[50,109,128,146]$. Hybrid models combine continuous and discrete methods in order to describe different aspects of the studied phenomenon. In this sense they are often considered in terms of multiscale modelling. In the last decade several different hybrid models of blood coagulation in flows were developed $[49,50,109,128,146]$.

A DPD-PDE hybrid method was considered in [49,137]. The DPD method was used to model blood plasma flow and platelets, while concentrations of proteins were described by diffusion-convection equations in order to model substances which induced platelet activation [49]. It was supposed that these agonists were produced by platelets in flow or in the platelet aggregate at the injury site. Once activated, the platelets can aggregate to each other or adhere to the vessel wall at the injury site. The main goal in [49] was to develop a model of platelet aggregation based on the assumption that platelet activation precedes platelet aggregation. The mechanisms of clot growth and growth arrest were studied in [137]. 

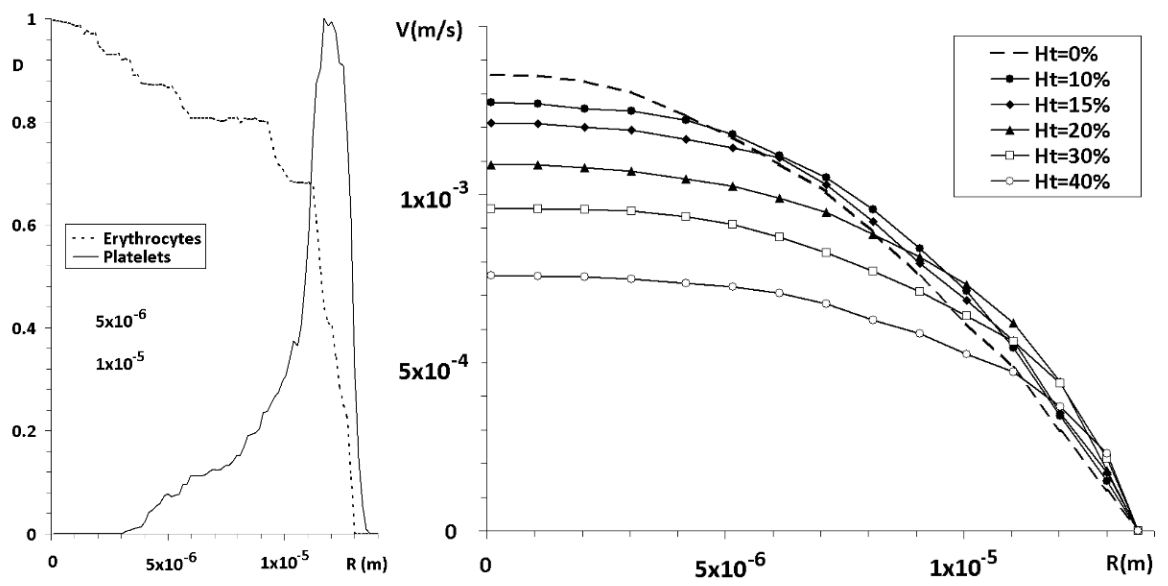

FiguRE 5. Averaged velocity profiles in simulations with erythrocytes and platelets. Velocity profiles are shown as functions of distance from the flow axis for the values of $H_{t}: 40 \%, 30 \%, 20 \%, 15 \%, 10 \%$ and without erythrocytes $\left(H_{t}=0\right)$. Reprinted with permission from [15].

\section{1D global flows in networks}

1D blood flow models have been used since the end of the last century. Their attractiveness is based on reasonable computational costs for studying regional, systemic and closed (global) circulation. Primary applications of these models are blood gases and drugs transport in the organism, blood flow redistribution under external or internal impacts such as physical activity or stimulation by medical devices, blood flow remodeling due to intravascular surgery such as stenting or shunting.

The primary physical assumption of $1 \mathrm{D}$ blood flow models is viscous incompressible fluid flow in the network of elastic tubes representing relatively large vessels. Therefore, at the most general level, the hydrodynamic part of the model is determined by the 3D Navier-Stokes equations and the elastic part of the model is described by deformable solid mechanics. Both parts are coupled, and the coupled fluidstructure interaction (FSI) problem is applicable to simulations of local flows in complicated regions (aortic arch, carotid arteries, large systemic arteries, etc.). The main drawbacks of 3D FSI models are complexity of numerical schemes, high computational cost and speculative boundary conditions. Moreover, uncertainty of model parameters and domain boundary reduce the significance of thoroughly computed 3D solution.

$1 \mathrm{D}$ flow equations are derived from averaging the $3 \mathrm{D}$ equations in a single vessel $[24,53]$. The following assumptions are conventional: the ratio of vessel diameter to its length is relatively small and the velocity field demonstrates Poiseuille profile at every cross-section, the vessel is represented by a thin elastic tube. The hemodynamic model of closed (global) circulation consists from 1D flow equations in separate vessels coupled by boundary conditions at junction points between the vessels and at the heart.

The computational domain is therefore the human vascular $1 \mathrm{D}$ network or its parts. The network can be generated on the basis of general anatomical data such as handbook of anatomical charts [21], anatomy 3D models [17] or patient-specific data [55]. The state-of-the-art technology for generation of personalized vascular network is illustrated in Fig.6. At the first stage one produces the segmentation of vascular system on the basis of CT or MRI data (Fig.6, left); at the second stage one extracts centerlines and radii of future tubular structures (Fig.6, center); at the third stage one generates the 3D graph representing the vascular network (Fig.6, right).

Further we discuss equations of global 1D hemodynamics including 1D flow equations, boundary conditions, and possible modifications due to physiological reactions. 

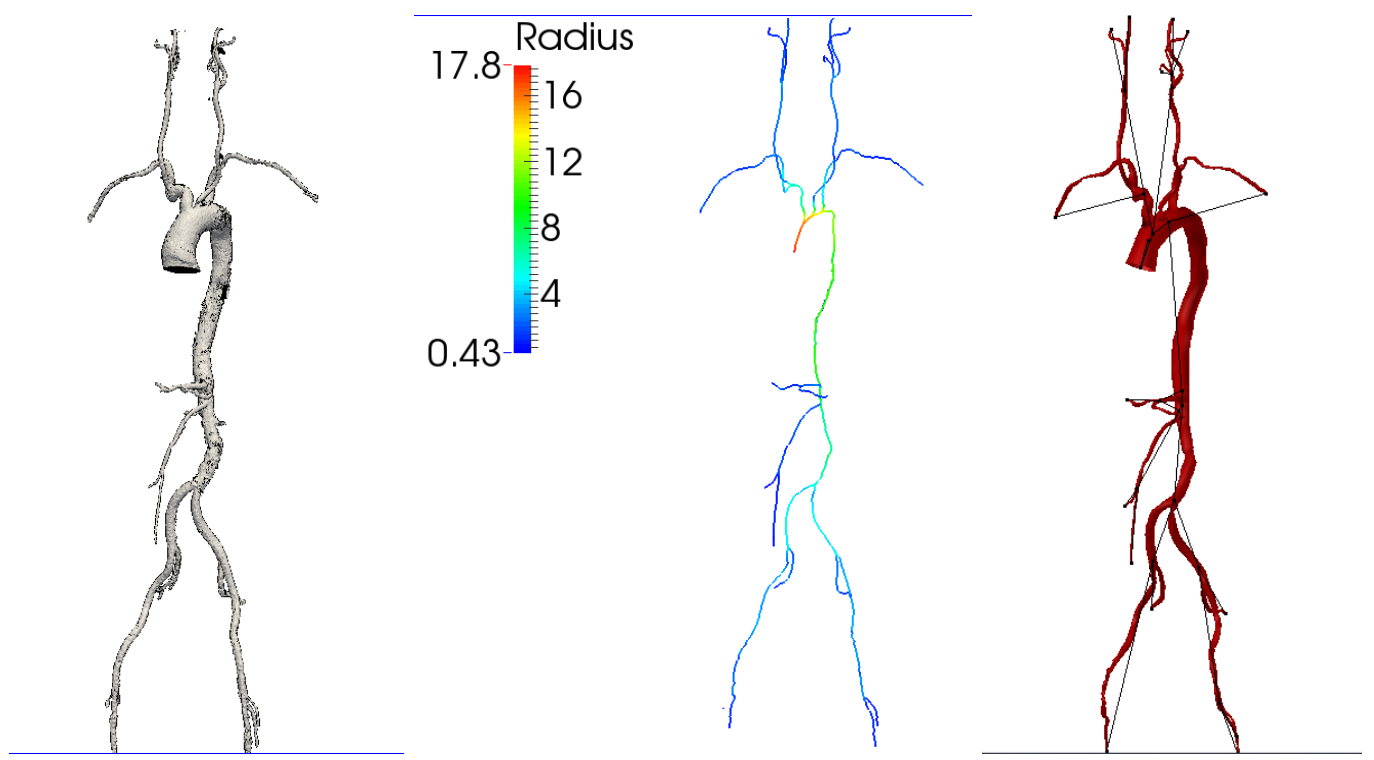

FiguRE 6. Stages of personalized network reconstruction: vascular segmentation (left), centerlines extraction (middle), 3D graph generation (right).

\subsection{D flow models}

The 1D flow in a separate vessel is described by the model of viscous incompressible fluid flow through an elastic tube. Denote by $t$ the time, by $x$ the coordinate along the vessel, by $A(t, x)$ the cross-section area of the vessel, by $u(t, x)$ and $p$ the averaged over the cross-section linear velocity and blood transmural pressure. Mass and momentum balance in $(A, u, p)$ variables correspond to equations

$$
\begin{array}{r}
\partial A / \partial t+\partial(A u) / \partial x=f_{A}, \\
\partial u / \partial t+\partial\left(u^{2} / 2+p / \rho\right) / \partial x=f_{u},
\end{array}
$$

where $\rho$ is the blood density (constant), $f_{A}$ is the mass source or sink per unit length, $f_{u}$ is the flow acceleration due to different forces (friction, gravity, circular force and other forces exerted on the fluid).

The right hand sides in equations (3.1)-(3.2) depend on the process to be simulated. For instance, the blood flow in an impermeable vessel out-of-external forces subjects to (3.1)-(3.2) with zero right hand sides. A blood source (e.g. transfusion) or sink (e.g. hemorrhage) can be formalized as

$$
f_{A}=\delta A,
$$

where $\delta$ is the source or sink intensity. The gravity acceleration can be accounted as

$$
f_{u}^{g}=g \sin \theta_{k},
$$

where $g$ is the gravity constant and $\theta$ is the angle between the vessel and the gravity field. This model was used in $[21,22]$ to simulate gravitational overload impact to the systemic circulation. The friction force [67] can be given by

$$
f_{u}^{f r}=-\frac{4 \pi \mu u}{A^{2}}\left(\kappa+\kappa^{-1}\right),
$$

where $\mu$ is the blood viscosity, $\kappa=A / A_{0}, A_{0}$ is the cross-section area of the unstressed vessel. Valve functioning in veins can be accounted within the $1 \mathrm{D}$ approach [123]

$$
f_{u}^{v l}=\left\{\begin{array}{l}
f_{u}^{f r}, u>0 \\
f_{\infty}^{f r}, u<0
\end{array}\right.
$$


where $f_{\infty}^{f r}=100 f_{f r}$ is the "infinite" resistance prohibitive for backward flows. We note that venous valve models extend from 3D [23,99] to 0D lumped parameters models [126].

Let $Q=A u$ denote the volumetric flow. The alternative formulation of mass and momentum balance in $(A, Q, p)$ variables may be given in the form $[2,53,75,91]$

$$
\begin{aligned}
\partial A / \partial t+\partial Q / \partial x & =f_{A}, \\
\partial Q / \partial t+\partial\left(Q^{2} / A\right) \partial x+A / \rho \partial p / \partial x+K_{R} Q / A & =f_{Q},
\end{aligned}
$$

where $K_{R}$ is the viscous flow resistance per unit length of the vessel. Similar formulation was also used in $[25,95,100,103,144]$. An interesting extension of this approach is given in $[33,41,54]$ where such formulation is extended to Korteweg-de Vries model.

\subsection{Elastic models of vessel wall}

In the 1D blood flow models, the conventional description of elastic properties of the vessel wall is provided by the pressure to cross-section area relationship $p(A)$. Straightforward approach to getting the relation $p(A)$ is precise simultaneous in vivo measurement of pressure and area at different times for typical vascular representatives (large, medium and small vessels at different localizations). Amenable technologies of intravascular pressure measurements make such approach impractical. On the other hand, in vitro mechanical study of extracted dead vascular tissues can not provide the material properties of the living tissue, not to speak of patient-specific material properties. Moreover, the dependence $p(A)$ is not solely mechanical: contraction of surrounding muscles, physiological features such as autoregulation, baroreceptor and chemoreceptors regulation, pathologies such as atherosclerosis, aneurysms and other systemic individual conditions may affect the results significantly. These observations make any derivation of $p(A)$ dependence subject to criticism.

The conventional wisdom is based on pure mechanical properties since this assumption allows experimental study of oscillating flows in collapsible tubes with passive elastic response. Qualitative analysis of physical experiments confirms that $p(A)$ function should be a monotone $S$-like curve. Such curve describes satisfactorily both circular and elliptic cross-section states $[27,58,92,104]$. In practice, $S$-like pressure-to-area dependence is often approximated by an analytical function. Correct elastic properties of both arteries and veins can be described by the following function [67]

$$
p(A)=\rho c_{0}^{2} f\left(A / A_{0}\right), f(\kappa)= \begin{cases}\exp (\kappa-1)-1, & \kappa>1 \\ \ln (\kappa), & \kappa \leqslant 1\end{cases}
$$

where $\kappa=A / A_{0}, c_{0}$ is the velocity of small disturbances propagation along the vessel (magnitude of $c_{0}$ is the measure of rigidity of the vessel wall). Different dependence is suggested in [91,92]

$$
f(\kappa) \sim \kappa^{m}-\kappa^{n}, m>0, n \in(-2,0) .
$$

Several $S$-like formulations and their linearized version were studied in [22]. Other dependencies $[2,53$, $75,82,100]$ provide good results for arteries.

The most advanced in silico studies of elastic vascular response use complex fiber reinforced material models $[61,90]$. In a simplified form, such models were used for studying the impacts of endovascular implants or atherosclerotic plaques to the $p(A)$ dependence [142]. Aneurysm model was developed in [82] by parameterising pressure-to-area function along the length of the diseased vessel. Other generalizations of the elastic vessel wall model are visco-elastic wall models $[2,16,25,102]$. In such models the $p(A)$ relationship depends on $\frac{\partial A}{\partial t}$ as well: $p(A)=F\left(A, \frac{\partial A}{\partial t}\right)$. Accounting vessel bending stiffness requires more arguments of function $F: p(A)=F\left(A, \frac{\partial A}{\partial t}, \frac{\partial^{2} A}{\partial t^{2}}, \frac{\partial^{2} A}{\partial x^{2}}\right)$. Such model is capable to explain the origin of Korotkoff sounds $[57,58]$.

Physical conditions and physiological reactions play a key role in cardiovascular system functioning. Biomedical applications are often related to transient regimes of average blood flow and/or regional flow 
redistribution. Flow variation implies variation of wall shear stress and permeability of vessel wall for $N O$ and, therefore, variation of concentration of $\mathrm{Ca}^{2+}$ and phosphorylated myosin that affects wall elasticity. A model of these processes [29] is self-adaptive to the flow so that it recovers the initial average shear stress. An alternative model [35] was applied to the cerebral circulation. The autoregulation can be incorporated in the 1D blood flow model [123] through the update of coefficient $c_{0}$ in (3.5) at the next cardiac cycle $n+1$ :

$$
\frac{c_{0}^{n+1}}{c_{0}^{n}}=\sqrt{\frac{\bar{p}_{n}}{\bar{p}_{n-1}},}
$$

where $\bar{p}_{n}$ is the average pressure within the cardiac cycle $n$. According to (3.7), the wall stiffness remains constant in quasi-periodic flow regimes in all parts of the network. In transient regimes, the wall stiffness is updated each cardiac cycle in every vessel. It regains to the initial values when the cardiovascular system returns to the initial quasi-periodic flow regime.

There exist other evidences of autoregulation which can be accounted in the 1D flow models. Baroreceptor regulation is taken into consideration through variation of the slope coefficient in $p(A)$ driven by mean pressure changes [72]. Autoregulation through oxygen concentration in cerebral vessels is considered in the terminal resistance adaptation model [3]. The model of regulation maintaining subsonic blood flow under gravitational overload was developed in [21]. The authors of [70] studied arteriolar autoregulation of cerebral vessels caused by altered gravity. For another review of autoregulation and neuroregulation models we refer to [122].

\subsection{Boundary conditions}

The 1D blood flow models require boundary conditions at vessels junctions and inlets and outlets of the vessel network.

For all vessels, boundary conditions should include compatibility conditions along characteristics of hyperbolic equations (3.1)-(3.2). It has been noted $[1,91,120,121,124,144]$ that for every vessel end point there exists one incoming and one outgoing characteristic which means subsonic flow regimes in almost all physiological cases. Thus, only one additional compatibility condition is required at every vessel end point.

The second conventional boundary condition at the junction of $N$ vessels is mass conservation

$$
\sum_{k=k_{1}, k_{2}, \ldots, k_{N}} \varepsilon_{k} A_{k}\left(t, \tilde{x}_{k}\right) u_{k}\left(t, \tilde{x}_{k}\right)=0
$$

where $\left\{k_{1}, \ldots, k_{N}\right\}$ are the indices of the incident vessels, $\varepsilon_{k}=1, \tilde{x}_{k}=0$ for incoming vessels, $\varepsilon_{k}=$ $-1, \tilde{x}_{k}=L_{k}$ for outgoing vessels. The other boundary conditions are $N$ pressure drop conditions

$$
p_{k}\left(A_{k}\left(t, \tilde{x}_{k}\right)\right)-p^{l}(t)=\varepsilon_{k} R_{k}^{l} A_{k}\left(t, \tilde{x}_{k}\right) u_{k}\left(t, \tilde{x}_{k}\right), k=k_{1}, k_{2}, \ldots, k_{N},
$$

or $N$ Bernoulli integral conservation conditions expressing the total pressure continuity $[3,16,82,91,100$, $102,120,121]$

$$
\frac{u_{k}^{2}}{2}+\frac{p_{k}\left(A_{k}\right)}{\rho}=P^{l}, k=k_{1}, k_{2}, \ldots, k_{N},
$$

where $p^{l}$ and $P^{l}$ are the pressure and the total pressure at the junction point with index $l$, respectively. A modification of this condition accounts the angle of the vessel bifurcation [52]. To summarize, at each vessel junction one imposes $2 N+1$ boundary conditions in terms of nonlinear algebraic equations which can be effectively reduced to $N+1$ nonlinear equations [141].

The conventional boundary condition at the heart outflow is a given function taken from literature or from patient measurements. Such approach does not result in the closed blood circulation model. In order to design the closed 1D network model, some authors suggest heart filling conditions at the junctions with pulmonary vein and vena cava as well as heart outflow conditions at the junctions with aorta and 
pulmonary artery through a lumped parameter model $[1,51,75,92,122,124]$. Alternative heart-vascular network coupling is discussed in [144].

Arteries network outlets and veins network inlets should be associated with the set of smaller unaccounted vessels belonging to microvascular regions. Flow in such vessels can not be described by the 1D flow models (3.1)-(3.4) due to large number of vessels, complex structure of microvascular networks and non-Newtonian blood rheology as discussed in section 1. An alternative 1D flow model was suggested recently for simulation of blood flow in microvascular network from a rat mesentery [103]. Another view on microvascular circulation is based on the dominance of diffusive processes over convective ones that suggests to consider filtration models in microvascular regions [68]. A simpler approach $[1,67,124]$ is to combine terminal arteries and veins to junction points where (3.8),(3.9) hold. Resistance coefficients $R_{k}^{l}$ are estimated from known pressure drop between arteries and veins. The larger vascular network, the smaller vessels are combined and the better accuracy of the method is.

Conventionally, 1D flow models treat microcirculation regions as specific boundary conditions. In the simplest case free outflow condition can be set at terminal arteries. More appropriate outflow boundary conditions are studied in [100]: selected parts of small arteries and microcirculation are modelled as structured trees whose root impedance can be evaluated from linearization of the governing equations. Many authors perform time-domain coupling of the 1D blood flow models with electric circuits (lumped parameters) 0D models $[4,16,36,73,80,87,92]$. The 0D models provide correct boundary conditions for global blood flow models reproducing specific reflections from natural microcirculatory bed. For more detailed review of this approach we refer to $[122,144]$.

The most papers report quite good correlation of the numerical solutions with general physiological data and 3D FSI simulations [145]. This implies that 1D blood flow modeling is the appropriate framework for regional and global blood flow simulations. Nowadays patient-specific modeling of 1D blood flows in networks is the challenge for the researchers. Personalized models will be the basis for in silico applications suitable in clinical practice.

\subsection{Numerical schemes}

From mathematical stand point, the 1D blood flow model is an algebraic-differential system composed from a set of hyperbolic equations in vessels and a set of algebraic equations at junctions and inlets/outlets of the vessel network. For its numerical solution diverse methods have been reported. The incomplete list contains Discontinuous Galerkin and Taylor-Galerkin methods [121], second-order local conservative Galerkin method [82,95], high-order Finite Volume method [91, 92], Finite Differences with artificial viscosity method [1], characteristic methods [52,67,120,124,141].

Although the blood flow is normally subsonic, external impacts or elasticity malfunction can produce transonic or even supersonic regimes. Monotonicity is important property for a robust numerical scheme. Examples of such schemes are the first order monotone characteristic method and the second order minimum oscillating method $[67,124,141]$. Another attractive feature of a numerical scheme is its low computational complexity. Fractional time steps implicit-explicit schemes [67,141] split computations into separate localized parts and thus are easily parallelized.

\subsection{Numerical example}

To demonstrate the applicability of global blood circulation models, we present the study of atherosclerotic plaque impact on hemodynamics by means of our numerical model [141-143].

We consider the systemic circle represented by two connected networks of arteries and veins. The vascular system consists of 341 vessels with anatomically adequate properties (lengths, diameters, elastic properties), the veins and arteries are connected in 162 points where boundary conditions (3.8),(3.9) are imposed. The arterial part of the network is presented in Fig.7. In each vessel we introduce a 1D uniform mesh and discretize the system (3.1)-(3.2) by the first order monotone characteristic method $[67,124]$. The equations are extended by a set of stiff ODEs which describe the heart functioning in terms of volume averaged model [124]. The system of stiff ODE's solved by A- and L-stable implicit third order 
Runge-Kutta method provides the boundary conditions at the heart inlet and outlet. The algebraicdifferential system (3.1),(3.2),(3.8),(3.9) coupled with a pressure-to-area relationship and appropriate boundary conditions at the heart and microcirculatory regions, is solved by the fractional time step scheme which splits computations in local independent parts (separate vessels and separate junction points). At the hyperbolic substep, we apply the explicit characteristic method for each vessel and control the time step by the stability restriction $\tau=0.9 s_{\max }$, where $s_{\max }=\max _{k, i}\left|\lambda_{k, i}\right| / h_{k}, h_{k}$ is the mesh size in vessel $k, \lambda_{k, i}$ is the largest (by magnitute) eigenvalue of the Jacobian for (3.1)-(3.2) at grid point $i$ of vessel $k$. At the algebraic substep, we apply the Newton method for a system of equations at each junction node. The system is composed of equations (3.8),(3.9) and a compatibility condition [124] along the characteristics of (3.1)-(3.2) leaving the integration domain for (3.1)-(3.2).

The impact of atherosclerotic plaque is accounted in the elastic wall model. Healthy (nonatherosclerotic) vessels are described by equation (3.5) providing feasible correlation with experimental curves. Atherosclerotic arteries are considered as three-layered cylindrical shells deformed by internal blood pressure. The inner and outer layers of the shell are the fibrous cap and the artery wall, respectively. The strains of the fibrous cap and the vessel wall are simulated by the fiber elastic model $[90,116]$. In the simplest version of the fiber axisymmetric model, the shell is represented by a set of circular ring fibers which resist only extension and compression as Neo-Hookean materials:

$$
\mathbf{F}=\frac{\partial}{\partial s}(T \boldsymbol{\tau}), \quad T=\mu\left(\left|\frac{\partial \mathbf{X}}{\partial s}\right|^{2}-\left|\frac{\partial \mathbf{X}}{\partial s}\right|^{-2}\right)
$$

Here $\mathbf{F}$ denotes the local force density, $T(s)$ denotes the fiber tension, $\boldsymbol{\tau}=\frac{\partial \mathbf{X}}{\partial s}\left|\frac{\partial \mathbf{X}}{\partial s}\right|^{-1}$ is the unit tangent vector, $\mathbf{X}(s)$ represents the position of the fiber points in space, Lagrange coordinate $s$ is the arc length of the fiber in the unstressed state. The lipid pool (intermediate shell) is imitated by a set of radial springs with nonlinear relation between the reaction force and the displacement $[141,143]$.

The pressure-to-area relation for the aterosclerotic artery is derived from the assumption of static equilibrium of the wall: the internal blood pressure is balanced by the elastic forces of the above fiberspring system generated by its displacement. Based on the displacements one can compute the cross section area $A$ as the reaction to any blood pressure. The recovery of the equilibrium state is obtained in the framework of its numerical approximation: finite difference discretization of (3.11) results in a system of nonlinear algebraic equations which has to be solved iteratively by the Newton method. We present our approach for the simplest axisymmetric lengthy plagues. The numerical fiber-spring model benefits straightforward generalizations with other types of fibers and thus can be extended to much wider class of plaque geometries.

In the experiment we assume that the left common carotid artery (No.5 in Fig.7) is damaged by the lengthy atherosclerotic plaque with 10\%,30\%,50\% and 100\% lumen. The coefficients of the elastic fiber-spring plaque model are taken from [143]. The velocity profiles in the external carotid continuation (No.94) and the arteries of the Willis circle (No.104,102) are shown in Fig.8. The most noticeable changes in velocity are in cases of plaques with lumen $30 \%$ and $10 \%$. In the small artery of Willis Circle (104) and on the continuation of the left external carotid artery (94) we can see significant decrease of blood velocity. The velocity decrease in small vessels leads to thrombosis and subsequent sharp pressure increase, whereas for the eye and the brain it results in oxygen deficiency and hence ischemia, hemorrhage, blindness and stroke. In the artery of Willis Circle (102) we observe the change of flow direction in case of plaque with lumen 10\%. Willis Circle blood recirculation is known to occur in some cases of stenosis, it provides physiologically optimal blood supply of the brain and the eyes.

To summarize, the 1D blood flow models can be used in analysis of the atherosclerosis impact on the regional blood supply. 

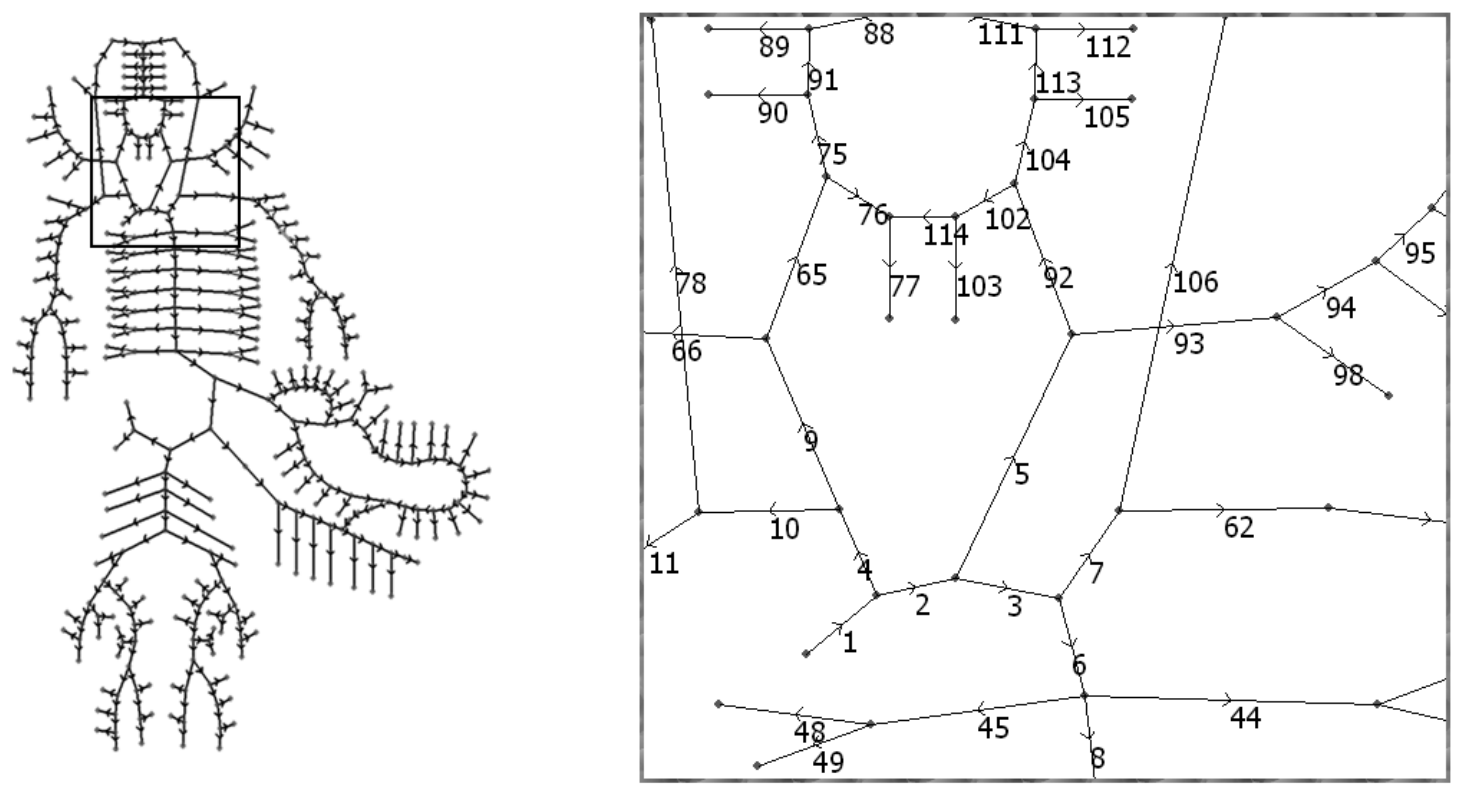

FiguRE 7. The systemic arterial network and its fragment with vessel indices.
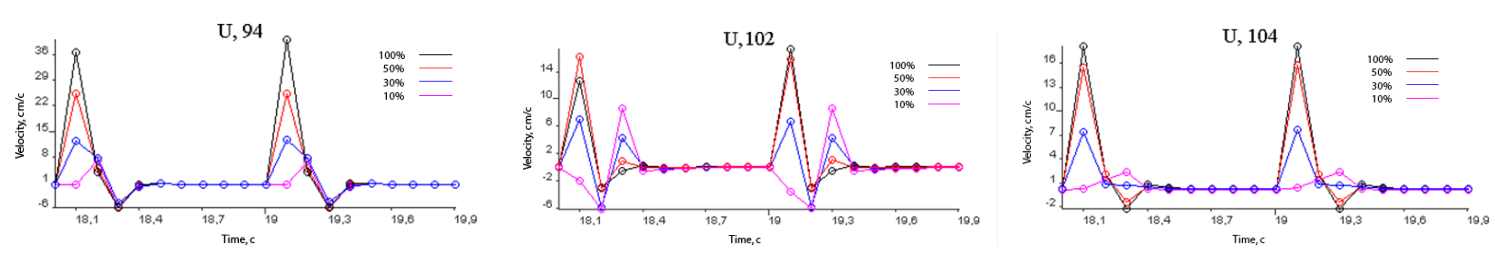

Figure 8. Velocity $(\mathrm{cm} / \mathrm{s})$ in vessels $94,102,104$ for different lumens. Healthy vessel corresponds to $100 \%$ lumen.

\section{Acknowledgements}

The work on section 3 was supported by the Russian Scientific Foundation Grant No. 14-31-00024. We thank Yuri Ivanov for images of Fig.6 and Tatiana Dobroserdova for simulations presented in Fig.8. The second author is thankful to FCT through CEMAT-Center for Computational and Stochastic Mathematics of Instituto Superior Técnico and the project EXCL/MAT-NAN/0114/2012.

\section{References}

[1] M.V. Abakumov, I.V. Ashmetkov, N.B. Esikova, V.B. Koshelev, S.I. Mukhin, N.V. Sosnin, V.F. Tishkin, A.P. Favorskij, A.B. Khrulenko. Strategy of mathematical cardiovascular system modeling. Matematicheskoe Modelirovanie, 12 (2000), no. $2,106-117$.

[2] J. Alastruey, A.W. Khir, K.S. Matthys, P. Segers, S.J. Sherwin, P.R. Verdonck, Kim H. Parker, J. Peiró. Pulse wave propagation in a model human arterial network: Assessment of 1-D visco-elastic simulations against in vitro measurements. Journal of Biomechanics, 44 (2011), 2250-2258. 
[3] J. Alastruey, S.M. Moore, K.H. Parker, T. David, J. Peiró, S.J. Sherwin. Reduced modelling of blood flow in the cerebral circulation: Coupling 1-D, $0-D$ and cerebral auto-regulation models. International journal for numerical methods in fluids, 56 (2008), no. 8, 1061-1067.

[4] J. Alastruey, K.H. Parker, J. Peiró, S.J. Sherwin. Lumped parameter outflow models for 1-D blood flow simulations: effect on pulse waves and parameter estimation. Communications in Computational Physics, 4 (2008), no. 2, 317-336.

[5] A.G. Alenitsyn, A.S. Kondratyev, I. Mikhailova, I. Siddique. Mathematical modeling of thrombus growth in microvessels. Journal of Prime Research in Mathematics, 4 (2008), 195-205.

[6] D. Alizadehrad, Y. Imai, K. Nakaaki, T. Ishikawa, T. Yamaguchi. Parallel simulation of cellular flow in microvessels using a particle method. Journal of Biomechanical Science and Engineering, 7 (2012), no. 1, 57-71.

[7] M.P. Allen, D.J. Tidesley. Computer Simulation of Liquids. Clarendon, Oxford, 1987.

[8] T. AlMomani, H.S. Udaykumar, J.S. Marshall, K.B. Chandran. Micro-scale dynamic simulation of erythrocyte-platelet interaction in blood flow. Annals of Biomedical Engineering, 36 (2008), no. 6, 905-920.

[9] M. Anand and K.R. Rajagopal. A shear-thinning viscoelastic fluid model for describing the flow of blood. Int. J. of Cardiovascular Medicine and Science, 4 (2004), no. 2, 59-68.

[10] M. Anand, K. Rajagopal, K.R. Rajagopal. A model for the formation, growth, and lysis of clots in quiescent plasma. A comparison between the effects of antithrombin III deficiency and protein C deficiency. J. Theor. Biol., 253 (2008), no. $4,725-738$.

[11] G. Astarita, G. Marrucci. Principles of Non-Newtonian Fluid Mechanics. McGraw Hill, 1974.

[12] P. Bagchi. Mesoscale simulation of blood flow in small vessels. Biophysical Journal, 92 (2007), no. 6, $1858-1877$. [PubMed: 17208982].

[13] H. A. Barnes. Thixotropy - a review. J. Non-Newtonian Fluid Mech., 70 (1997), 1-33.

[14] N. M. Bessonov, S.F. Golovashchenko, V. Volpert. Numerical modelling of contact elastic-plastic flows. Math. Model. Nat. Phenom., 4 (2008), no. 1, 44-87.

[15] N. Bessonov, E. Babushkina, S.F. Golovashchenko, A. Tosenberger, F. Ataullakhanov, M. Panteleev, A. Tokarev, V. Volpert. Numerical modelling of cell distribution in blood flow. Math. Model. Nat. Phenom., 9 (2014), no. 6, 69-84.

[16] P.J. Blanco, R.A. Feijóo. A 3D-1D-OD Computational model for the entire cardiovascular system. Computational Mechanics, eds. E.Dvorking, M. Goldschmit, M. Storti, XXIX (2010), 5887-5911.

[17] P.J. Blanco, S.M. Watanabe, M.A.R.F. Passos, P.A. Lemos, R.A. Feijóo. An anatomically detailed arterial network model for one-dimensional computational hemodynamics. IEEE Transaction on Biomedical Engineering, 62 (2015), no. 2, 736-753.

[18] T. Bodnar, K. Rajagopal, A. Sequeira. Simulation of the three-dimensional flow of blood using a shear-thinning viscoelastic fluid model. Math. Model. Nat. Phenom., 6 (2011), no. 5, 1-24.

[19] T. Bodnar, A. Sequeria. Numerical simulation of the coagulation dynamics of blood. Computational and Mathematical Methods in Medicine, 9 (2008), no. 2, 83-104.

[20] C. Bui, V. Lleras, O. Pantz. Dynamics of red blood cells in 2d. ESAIM: Proc., 28 (2009), 182-194.

[21] A. Ya. Bunicheva, M. A. Menyailova, S. I. Mukhin, N. V. Sosnin, A. P. Favorskii. Studying the influence of gravitational overloads on the parameters of blood flow in vessels of greater circulation. Mathematical Models and Computer Simulations, 5 (2013), no. 1, 81-91.

[22] A.Ya. Bunicheva, S.I. Mukhin, N.V. Sosnin, A.P. Favorskii. Numerical experiment in hemodynamics. Differential Equations, 40 (2004), no. 7, 984-999.

[23] G.A. Buxton, N. Clarke. Computational phlebology: the simulation of a vein valve. Journal of Biological Physics, 32 (2006), no. 6, 507-521.

[24] S. Canić, E.H. Kim. Mathematical analysis of the quasilinear eects in a hyperbolic model blood ow through compliant axi-symmetric vessels. Mathematical Methods in the Applied Sciences, 26 (2003), 1161-1186.

[25] S. C̆anić, J. Tambača, G. Guidoboni, A. Mikelić, C.J. Hartley, A. Rosenstrauch. Modeling viscoelastic behaviour of arterial walls and their interaction with pulsatile blood flow. SIAM Journal of Applied Mathematics, 67 (2006), no. 1, 164-193.

[26] C. G. Caro, T. J. Pedley, R. C. Schroter, W. A. Seed. The Mechanics of the Circulation. Oxford University Press, 1978.

[27] C.G. Caro, T.J. Pedley, R.C. Schroter, W.A. Seed. The Mechanics of the Circulation. 2nd Edition, Cambridge University Press, 2012.

[28] S. E. Charm, G. S. Kurland. Blood Flow and Microcirculation. John Wiley \& Sons, 1974.

[29] I.L. Chernyavsky, N.A. Kudryashov. A Mathematical model for autoregulation of the arterial lumen by endotheliumderived relaxing factor. Advanced Science Letters, 1 (2008), no. 2, 226-230.

[30] S. Chien, S. Usami, R.J. Dellenback, M.I. Gregersen. Shear dependence of effective cell volume as a determinant of blood viscosity. Science, 168 (1970), 977-979.

[31] S. Chien, R. G. King, R. Skalak, S. Usami, and A. L. Copley. Viscoelastic properties of human blood and red cell suspensions. Biorheology, 12 (1975), 341-346.

[32] Y. I. Cho and K. R. Kensey. Effects of the non-Newtonian viscosity of blood on flows in a diseased arterial vessel. Part I: Steady flows. Biorheology, 28 (1991), 241-262.

[33] E. Crepeau, M. Sorine. A reduced model of pulsatile flow in an arterial compartment. Chaos Solitons \& Fractals, 34 (2007), no. 2, 594-605.

[34] L.M. Crowl, A.L. Fogelson. Computational model of whole blood exhibiting lateral platelet motion induced by red blood cells. Int. J. Numer. Method Biomed. Eng., 26 (2010), no. 3-4, 471-487. 
[35] T. David, S. Alzaidi, H. Farr. Coupled autoregulation models in the cerebro-vasculature. Journal of Engineering Mathematics, 64 (2009), 403-415.

[36] A. DiCarlo, P. Nardinocchi, G. Pontrelli, L. Teresi. A heterogeneous approach for modelling blood flow in an arterial segment. Simulations in Biomedicine V, WIT Press, 69-78, 2003.

[37] L. Dintenfass. Blood Microrheology -Viscosity Factors in Blood Flow, Ischaemia and Thrombosis. Butterworth, 1971.

[38] L. Dintenfass. Blood Viscosity, Hyperviscosity and Hyperviscosaemia. MTP Press Limited, 1985.

[39] M.M. Dupin, I. Halliday, C.M. Care, L. Alboul, L.L. Munn, Modeling the flow of dense suspensions of deformable particles in three dimensions, Physical Review E, 75 (2007), 066707.

[40] W. Dzwinel, K. Boryczko, D.A. Yuen. Modeling mesoscopic fluids with discrete-particles methods. Algorithms and results. In: Spasic AM, Hsu JP (eds) Finely Dispersed Particles: Micro-, Nano-, and Atto-Engineering. Taylor \& Francis, CRC Press, 715-778.

[41] A. Elgarayhi, E.K. El-Shewy, A.A. Mahmoud, A.A. Elhakem. Propagation of nonlinear pressure waves in blood. ISRN Computational Biology, 2013, Article ID 436267.

[42] E. A. Evans, R. M. Hochmuth. Membrane viscoelasticity. Biophys. J., 16 (1976), no. 1, 111.

[43] D. Fedosov, B. Caswell, G.E. Karniadakis, General coarse-grained red blood cell models: I. Mechanics, 2009, arXiv:0905.0042 [q-bio.CB].

[44] D. Fedosov, B. Caswell, G.E. Karniadakis, A multiscale red blood cell model with accurate mechanics, rheology, and dynamics, Biophysical Journal, 98 (2010), 2215-2225.

[45] D.A. Fedosov, Multiscale Modeling of Blood Flow and Soft Matter, PhD dissertation at Brown University, (2010).

[46] D.A. Fedosov, H. Lei, B. Caswell, S. Suresh, G.E. Karniadakis, Multiscale modeling of red blood cell mechanics and blood flow in malaria. PLoS Computational Biology, 7 (2011), no. 12, e1002270.

[47] D.A. Fedosov, H. Noguchi, G. Gompper. Multiscale modeling of blood flow: from single cells to blood rheology. Biomech. Model. Mechanobiol., 13 (2014), 239-258.

[48] D.A. Fedosov, I.V. Pivkin, G.E. Karniadakis, Velocity limit in DPD simulations of wall-bounded flows. J. Comp. Phys., 227 (2008) 2540-2559.

[49] N. Filipovic, M. Kojic, A. Tsuda. Modelling thrombosis using dissipative particle dynamics method. Phil. Trans. R. Soc. A, 366 (2008), 3265-3279.

[50] A.L. Fogelson. Cell-based models of blood clotting. Single-Cell-Based Models in Biology and Medicine (ed. by A.R.A. Anderson, M.A.J. Chaplain, K.A. Rejniak), Mathematics and Biosciences in Interaction, p. 234-169, Birkhäuser Verlag Basel, 2007.

[51] L. Formaggia, D. Lamponi, M. Tuveri, A. Veneziani. Numerical modeling of $1 D$ arterial networks coupled with a lumped parameters description of the heart. Computer Methods in Biomechanics and Biomedical Engineering, 9 (2006), no. 5, $273-288$.

[52] L. Formaggia, D. Lamponi, A. Quarteroni. One-dimensional models for blood flow in arteries. Journal of Engineering Mathematics, 47 (2003), 251-276.

[53] L. Formaggia, A. Quarteroni, A. Veneziani. Cardiovascular mathematics. Vol. 1. Springer, Heidelberg, 2009.

[54] T.K. Gaik, H. Demiray. Forced Korteweg-de Vries-Burgers equation in an elastic tube filled with a variable viscosity fluid. Chaos Solitons \& Fractals, 38 (2008), 1134-1145.

[55] T. Gamilov, Y. Ivanov, P. Kopylov, S. Simakov, Y. Vassilevski. Patient specific haemodynamic modeling after occlusion treatment in leg. Math. Model. Nat. Phenom., 9 (2014), no. 6, 85-97.

[56] H.L. Goldsmith, V.T. Turitto. Rheological aspects of thrombosis and haemostasis: basic principles and applications. Thrombosis and Haemostasis, 55 (1986), no. 3, 415-435.

[57] S. S. Grigorjan, Y.Z. Saakjan, A. K. Tsaturjan. On the mechanisms of generation of Korotkoff sounds. Doklady of Academy of Science of the SSSR, 251 (1980), 570-574 (in Russian).

[58] S.S. Grigorjan, Y.Z. Saakjan, A.K. Tsatutjan. To the theory of Korotkoff method. Biomechanics, (1984), no. 15-16, 54-75.

[59] R.D. Groot, P.B. Warren, Dissipative particle dynamics: bridging the gap between atomistic and mesoscopic simulation. J. Chem. Phys., 107 (1997), no. 11, 4423-4435.

[60] R.D. Guy, A.L. Fogelson, J.P.Keener. Fibrin gel formation in a shear flow. Math. Med. Biol. 24 (2007), no. 1, $111-130$.

[61] G.A. Holzapfel, T.C. Gasser, R.W. Ogden. A new constitutive framework for arterial wall mechanics and a comparative study of material models. Journal of Elasticity, 61 (2000), 1-48.

[62] S.M. Hosseini, J.J. Feng. A particle-based model for the transport of erythrocytes in capillaries. Chem. Eng. Sci., 64 (2009), 4488-4497.

[63] Y. Imai, H. Kondo, T. Ishikawa, C.T. Lim, T. Yamaguchi. Modeling of hemodynamics arising from malaria infection. Journal of Biomechanics, 43 (2010), 1386-1393.

[64] Y. Imai, K. Nakaaki, H. Kondo, T. Ishikawa, C.T. Lim, T. Yamaguchi. Margination of red blood cells infected by Plasmodium falciparum in a microvessel. Journal of Biomechanics, 44 (2011), 1553-1558.

[65] M. Karttunen, I. Vattulainen, A. Lukkarinen. A Novel Methods in Soft Matter Simulations. Springer, Berlin, 2004.

[66] J. Keener, J. Sneyd. Mathematical Physiology. II: Systems Physiology. Springer, 2nd edition, 2008.

[67] A.S. Kholodov. Some dynamical models of external breathing and haemodynamics accounting for their coupling and substance transport. Computer Models and Medicine Progress, Nauka, Moscow, 127-163, 2001 (in Russian).

[68] A.S. Kholodov, A.V. Evdokimov, S.S. Simakov. Numerical simulation of peripheral circulation and substance transfer with $2 D$ models. Mathematical biology: recent trends, eds. P. Chandra, R. Kumar, 22-29, 2006. 
[69] S. Kim, Y.I . Cho, A. H. Jeon, B. Hogenauer, K. R. Kensey. A new method for blood viscosity measurement. J. Non-Newtonian Fluid Mech., 94 (2000), 47-56.

[70] C.S. Kim, C. Kris, D. Kwak. Numerical models of human circulatory system under altered gravity: brain circulation. AIAA Paper No. 2004-1092, AIAA 42nd Aerospace Sciences Meeting and Exhibit, Reno, NV, January 2004.

[71] J.F. Koleski, E.C. Eckstein. Near wall concentration profiles of 1.0 and $2.5 \mu \mathrm{m}$ beads during flow of blood suspensions, Trans. Ann. Soc. Intern. Organs, 37 (1991), 9-12.

[72] V. Koshelev, S. Mukhin, T. Sokolova, N. Sosnin, A. Favorski. Mathematical modelling of cardio-vascular hemodynamics with account of neuroregulation. Matematicheskoe Modelirovanie, 19 (2007), no. 3, 15-28 (in Russian).

[73] W. Kroon, W. Huberts, M. Bosboom, F. van de Vosse. A numerical method of reduced complexity for simulating vascular hemodynamics using coupled OD lumped and $1 D$ wave propagation models. Computational and Mathematical Methods in Medicine, (2012), Article ID 156094.

[74] P.W. Kuchel, E.D. Fackerell. Parametric-equation representation of biconcave erythrocytes. Bulletin of Mathematical Biology, 61 (1999), 209-220.

[75] I. Larrabidea, P.J. Blanco, S.A. Urquiza, E.A. Dari, M.J. Véneref, N.A. de Souza e Silvac, R.A. Feijóo. HeMoLab hemodynamics modelling laboratory: an application for modelling the human cardiovascular system. Computers in Biology and Medicine, 42 (2012), 993-1004.

[76] M. B. Lawrence, T. A. Springer. Leukocytes roll on a selectin at physiological flow rates: distinction from and prerequisite for adhesion through integrins. Cell, 65 (1991), 859-873.

[77] R.C. Leif, J. Vinograd, The Distribution of Buoyant Density of Human Erythrocytes in Bovine Albumin Solutions, Proc. Natl. Acad. Sci. USA, 51 (1964), no. 3, 520-528.

[78] S. Leibler, A.C. Maggs, Simulation of shape changes and adhesion phenomena in an elastic model of erythrocytes. Proc. Natl. Acad. Sci. USA, 87 (1990), 6433-6435.

[79] D. Liepsch, St. Moravec. Pulsatile flow of non-Newtonian fluid in distensible models of human arteries. Biorheology, 21 (1984), 571-586.

[80] K. Logana, R. Balossino, F. Migliavacca, G. Pennati, E.L. Bove, M.R. Leval, G. Dubini, Multiscale modeling of the cardiovascular system: application to the study of pulmonary and coronary perfusion in the univentricular circulation. Journal of Biomechanics, 38 (2005), no. 5, 1129-1141.

[81] L. Lopez, I.M. Duck, W.A. Hunt. On the shape of the erythrocyte. Biophys. J., 8 (1968), no. 11, $1228-1235$.

[82] K. Low, R. van Loon, I. Sazonov, R.L.T. Bevan, P. Nithiarasu. An improved baseline model for a human arterial network to study the impact of aneurysms on pressure-flow waveforms. International Journal of Numerical Methods in Biomedical Engineering, 28 (2012), 1224-1246.

[83] G. D. O. Lowe, Ed. Clinical Blood Rheology, Vol. I and II. CRC Press, Boca Raton, Florida, 1998.

[84] J.L. McWhirter, H. Noguchi, G. Gompper. Flow-induced clustering and alignment of vesicles and red blood cells in microcapillaries. PNAS, 106 (2009), no. 15, 6039-6043.

[85] E. W. Merrill, E. R. Gilliland, G. Cokelet, H. Shin, A. Britten, R. E. Wells, Jr.. Rheology of human blood, near and at zero flow. Effects of temperature and hematocrit level. Biophys. J., 3 (1963), 199-213.

[86] E. W. Merrill, G. C. Cokelet, A. Britten, R. E. Wells. Non-Newtonian rheology of human blood. Effect of fibrinogen deduced by subtraction. Circulat. Res., 13 (1963), 48-55.

[87] V. Milisić, A. Quarteroni. Analysis of lumped parameter models for blood flow simulations and their relation with $1 D$ models. ESAIM: Mathematical Modelling and Numerical Analysis, 38 (2004), no. 4, 613-632.

[88] N. Mohandas, P.G. Gallagher, Red cell membrane: past, present, and future. Blood, 112 (2008)m 3939-3948.

[89] P.C. F. Moller, J. Mewis, D. Bonn. Yield stress and thixotropy: on the difficulty of measuring yield stress in practice. Soft Matter, 2 (2006), 274-288.

[90] Y. Mori, C. Peskin. A universal programmable fiber architecture for the representation of a general incompressible linearly elastic material as a fiber-reinforced fluid. Advances in Applied Mathematics, 43 (2009), no. 1, 75-100.

[91] L.O. Müller, C. Parés, E. Toro. Well-balanced high-order numerical schemes for one-dimensional blood flow in vessels with varying mechanical properties. Journal of Computational Physics, 242 (2013), 53-85.

[92] L.O. Müller, E. Toro. A global multiscale mathematical model for the human circulation with emphasis on the venous system. International Journal for Numerical Methods in Biomedical Engineering, 30 (2014), no. 7, 681-725.

[93] L.L. Munn, M.M. Dupin, Blood Cell Interactions and Segregation in Flow. Annals of Biomedical Engineering, 36 (2008), no. 4, 534-544.

[94] S. Muñoz San Martín, J.L. Sebastián, M. Sancho1, G. Álvarez. Modeling human erythrocyte shape and size abnormalities. arXiv:q-bio/0507024 [q-bio.QM], 14 Jul 2005.

[95] J.P. Mynard, P. Nithiarasu. A $1 D$ arterial blood flow model incorporating ventricular pressure, aortic valve and regional coronary flow using the locally conservative Galerkin ( $L C G$ ) method. Communications in Numerical Methods in Engineering, 24 (2008), no. 5, 367-417.

[96] Q. D. Nguyen, D. V. Boger. Measuring the flow properties of yield stress fluids. Annual Reviews, 24 (1992), 47-88.

[97] H. Noguchi, G. Gompper. Shape transitions of fluid vesicles and red blood cells in capillary flows. PNAS, 102 (2005), no. 40, 14159-14164.

[98] D. Obrist, B. Weber, A. Buck, P. Jenny. Red blood cell distribution in simplified capillary networks, Phil. Trans. R. Soc. A, 368 (2010), doi: 10.1098/rsta.2010.0045.

[99] T. Ohashi, H. Liu, T. Yamaguchi. Computational fluid dynamic simulation of the flow through venous valve. In: Clinical Application of Computational Mechanics to the Cardiovascular System, 186-189, Springer, 2000. 
[100] M.S. Olufsen, C.S. Peskin, W.Y. Kim, E.M. Pedersen, A. Nadim, J. Larsen. Numerical simulation and experimental validation of blood flow in arteries with structured-tree outflow conditions. Annals of Biomedical Engineering, 28 (2000), 1281-1299.

[101] R. G. Owens. A new microstructure-based constitutive model for human blood, J. Non -Newtonian Fluid Mech., 14 (2006), 57-70.

[102] E. Ozawa, K. Bottom, X. Xiao R.D. Kamm. Numerical simulation of enhanced external counterpulsation. Annals of Biomedical Engineering, 29 (2001), 284-297.

[103] Q. Pan, R. Wang, B. Reglin, G. Cai, J. Yan, A.R. Pries, G. Ning. A one-dimensional mathematical model for studying the pulsatile flow in microvascular networks. Journal of Biomedical Engineering, 136 (2014), no. 1, 011009.

[104] T.J. Pedley, X.Y. Luo. Modelling flow and oscillations in collapsible tubes. Theoretical and Computational Fluid Dynamics, 10 (1998), 277-294.

[105] D. Pinho, A. Pereira, R. Lima, T. Ishikawa, Y. Imai, T. Yamaguchi. Red blood cell dispersion in 100 mm glass capillaries: the temperature effect. C.T. Lim and J.C.H. Goh (Eds.), WCB 2010, IFMBE Proceedings, 31 (2010), 1067-1070.

[106] E. Pinto, B. Taboada, R. Rodrigues, V. Faustino, A. Pereira, R. Lima. Cell-free layer (CFL) analysis in a polydimethysiloxane (PDMS) microchannel: a global approach. WebmedCentral Biomedical Engineering, 4 (2013), 8, WMC004374.

[107] I.V. Pivkin, G.E. Karniadakis, Accurate coarse-grained modeling of red blood cells. Physical Review letters, 101 (2008), 118105.

[108] I.V. Pivkin, G.E. Karniadakis. A new method to impose no-slip boundary conditions in dissipative particle dynamics. J. Comp. Phys., 207 (2005), 114-128.

[109] I.V. Pivkin, P.D. Richardson, G. Karniadakis. Blood flow velocity effects and role of activation delay time on growth and form of platelet thrombi. PNAS, 103 (2006), 17164-17169.

[110] A. S. Popel, P. C. Johnson. Microcirculation and hemorheology. Annu. Rev. Fluid Mech., 37 (2005), 43-69.

[111] C. Pozrikidis. Modeling and Simulation of Capsules and Biological Cells, Chapman \& Hall/CRC, 2003.

[112] D. Quemada. Rheology of concentrated disperse systems III. General features of the proposed non-Newtonian model. Comparison with experimental data. Rheological Acta, 17 (1978), 643-653.

[113] K.R. Rajagopal, A.R. Srinivasa. A thermodynamic frame work for rate type fluid models. Journal of Non-Newtonian Fluid Mechanics, 80 (2000), 207-227.

[114] A.M. Robertson, A. Sequeira, M.V. Kameneva. Hemorheology. In G.P. Galdi, R. Rannacher, A.M. Robertson, S. Turek (Eds.) Hemodynamical Flows: Modeling, Analysis and Simulation. (Oberwolfach Seminars), Birkhäuser Verlag, 37, 63-120, 2008.

[115] M.C. Roco, editor. Particulate Two-Phase Flow. Series in Chemical Engineering. Butterworth-Heinemann Publ., 1993.

[116] M. Rosar, C. Peskin. Fluid flow in collapsible elastic tubes: a three-dimensional numerical model. New York Journal of Mathematics, 7 (2001), 281-302.

[117] U.D. Schiller. Dissipative Particle Dynamics. A Study of the Methodological Background. Diploma thesis at Faculty of Physics University of Bielefeld, 2005.

[118] H. Schmid-Schönbein, R. E. Wells. Rheological properties of human erythrocytes and their influence upon anomalous viscosity of blood. Physiology Rev., 63 (1971), 147-219.

[119] G. W. Scott-Blair. An equation for the flow of blood, plasma and serum through glass capillaries. Nature, 183 (1959), 613-614.

[120] S. Sherwin, V. Franke, J. Peiró, K. Parker. One-dimensional modelling of a vascular network in space-time variables. Journal of Engineering Mathematics, 47 (2003), 217-250.

[121] S.J. Sherwin, L. Formaggia, J. Peiró, V. Franke. Computational modelling of $1 D$ blood flow with variable mechanical properties and its application to the simulation of wave propagation in the human arterial system. International Journal for Numerical Methods in Fluids, 43 (2003), 673-700.

[122] Y. Shi, P. Lawford, R. Hose. Review of zero-D and 1-D models of blood flow in the cardiovascular system. BioMedical Engineering Online, 10:33 (2011), doi:10.1186/1475-925X-10-33.

[123] S.S. Simakov, T.M. Gamilov, Y.N. Soe. Computational study of blood flow in lower extremities under intense physical load. Russian Journal of Numerical Analysis and Mathematical Modelling, 28 (2013), no. 5, 485-504.

[124] S.S. Simakov, A.S. Kholodov. Computational study of oxygen concentration in human blood under low frequency disturbances. Mathematical Models and Computer Simulations, 1 (2009), 283-295.

[125] R. Skalak, A. Tozeren, R. Zarda, S. Chein. Strain energy function of red blood cell membranes. Biophysical Journal 13 (1973), no. 3, 245-264 [PubMed: 4697236].

[126] M.F. Snyder, V.C. Rideout. Computer simulation studies of the venous circulation. IEEE Transactions on Bio-Medical Engineering, BME-16 (1969) no. 4, 325-334.

[127] S. Suresh, J. Spatz, J. P. Mills, A. Micoulet, M. Dao, C. T. Lim, M. Beil, T. Seufferlein. Connections between single-cell biomechanics and human disease states: gastrointestinal cancer and malaria. Acta Biomaterialia, 1 (2005), 15-30.

[128] C.R. Sweet, S. Chatterjee, Z. Xu, K. Bisordi, E.D. Rosen, M. Alber. Modelling platelet - blood flow interaction using the subcellular element Langevin method. J. R. Soc. Interface, 8 (2011), 1760-1771.

[129] G. B. Thurston. Viscoelasticity of human blood. Biophys. J., 12 (1972), 1205-1217.

[130] G.B. Thurston. Non-Newtonian viscosity of human blood: Flow induced changes in microstructure. Biorheology, 31 (1994), no. 2, 179-192. 
[131] G. B. Thurston. Viscoelastic properties of blood and blood analogs. Advances in Hemodynamics and Hemorheology, 1 (1996), 1-30.

[132] A.A. Tokarev, A.A. Butylin, F.I. Ataullakhanov. Platelet adhesion from shear blood flow is controlled by near-wall rebounding collisions with erythrocytes. Biophys. J., 100 (2011), no. 4, 799-808.

[133] A.A. Tokarev, A.A. Butylin, F.I. Ataullakhanov. Platelet transport and adhesion in shear blood flow: the role of erythrocytes. Computer Research and Modeling, 4 (2012), no. 1, 185-200 (Russian).

[134] A.A. Tokarev, A.A. Butylin, E.A. Ermakova, E.E. Shnol, G.P. Panasenko, F.I. Ataullakhanov. Finite platelet size could be responsible for platelet margination effect. Biophysical Journal, 101 (2011), 1835-1843.

[135] A. Tokarev, I. Sirakov, G. Panasenko, V. Volpert, E. Shnol, A. Butylin, F. Ataullakhanov. Continuous mathematical model of platelet thrombus formation in blood flow. Russian Journal of Numerical Analysis and Mathematical Modelling, 27 (2012), no. 2, 192-212.

[136] A. Tosenberger, V. Salnikov, N. Bessonov, E. Babushkina, V. Volpert. Particle dynamics methods of blood flow simulations. Math. Model. Nat. Phenom., 6 (2011), no. 5, 320-332.

[137] A. Tosenberger, F. Ataullakhanov, N. Bessonov, M. Panteleev, A. Tokarev, V. Volpert. Modelling of thrombus growth in flow with a DPD-PDE method. Journal of Theoretical Biology, 337 (2013), 30-41.

[138] K. Tsubota, S. Wada. Elastic force of red blood cell membrane during tank-treading motion: Consideration of the membrane's natural state. International Journal of Mechanical Sciences, 52 (2010), 356-364.

[139] K. Tsubota, S. Wada, H. Kamada, Y. Kitagawa, R. Lima, T. Yamaguchi. A particle method for blood flow simulation, application to flowing red blood cells and platelets. Journal of the Earth Simulator, 5 (2006), 2-7.

[140] F. J. Walburn, D. J. Schneck. A constitutive equation for whole human blood. Biorheology, 13 (1976), 201-210.

[141] Yu. Vassilevskii, S. Simakov, V. Salamatova, Yu. Ivanov, T. Dobroserdova. Numerical issues of modelling blood flow in networks of vessels with pathologies. Russian Journal of Numerical Analysis and Mathematical Modelling, 26 (2011), no. 6, 605-622.

[142] Y. Vassilevski, S. Simakov, V. Salamatova, Y. Ivanov, T. Dobroserdova. Blood flow simulation in atherosclerotic vascular network using fiber-spring representation of diseased wall. Math. Model. Nat. Phenom., 6 (2011), no. 5, 333-349.

[143] Y. Vassilevski, S. Simakov, V. Salamatova, Y. Ivanov, T. Dobroserdova. Vessel wall models for simulation of atherosclerotic vascular networks. Math. Model. Nat. Phenom., 6 (2011), no. 7, 82-99.

[144] F.N. van de Vosse, N. Stergiopulos. Pulse wave propagation in the arterial tree. Annual Review of Fluid Mechanics, 43 (2011), 467-499.

[145] N. Xiao, J. Alastruey-Arimon, C.A. Figueroa. A systematic comparison between $1 D$ and $3 D$ hemodynamics in compliant arterial models. International Journal for Numerical Methods in Biomedical Engineering. 30 (2014), no. 2, $204-231$.

[146] Z. Xu, N. Chen, M.M. Kamocka, E.D. Rosen, M. Alber. A multiscale model of thrombus development. J. R. Soc. Interface, 5 (2008), 705-722.

[147] C. Yeh, A.C. Calvez, E.c. Eckstein. An estimated shape function for drift in a platelet-transport model. Biophysical Journal, 67 (1994), 1252-1259.

[148] C. Yeh, E.C. Eckstein. Transient lateral transport of platelet-sized particles in flowing blood suspensions. Biophysical Journal, 66 (1994), 1706-1716.

[149] K.K. Yeleswarapu, M.V. Kameneva, K. R. Rajagopal, J. F. Antaki. The flow of blood in tubes: Theory and experiment. Mechanics Research Communications, 25 (1998), no. 3, 257-262.

[150] J. Zhang, P.C. Johnson, A.S. Popel. Effects of erythrocyte deformability and aggregation on the cell free layer and apparent viscosity of microscopic blood flows. Microvasc Res., 77 (2009), 265-272. 